\title{
RANDOM BIT QUADRATURE AND APPROXIMATION OF DISTRIBUTIONS ON HILBERT SPACES
}

\author{
MICHAEL B. GILES, MARIO HEFTER, LUKAS MAYER, AND KLAUS RITTER
}

\begin{abstract}
We study the approximation of expectations $\mathrm{E}(f(X))$ for Gaussian random elements $X$ with values in a separable Hilbert space $H$ and Lipschitz continuous functionals $f: H \rightarrow \mathbb{R}$. We consider restricted Monte Carlo algorithms, which may only use random bits instead of random numbers. We determine the asymptotics (in some cases sharp up to multiplicative constants, in the other cases sharp up to logarithmic factors) of the corresponding $n$-th minimal error in terms of the decay of the eigenvalues of the covariance operator of $X$. It turns out that, within the margins from above, restricted Monte Carlo algorithms are not inferior to arbitrary Monte Carlo algorithms, and suitable random bit multilevel algorithms are optimal. The analysis of this problem leads to a variant of the quantization problem, namely, the optimal approximation of probability measures on $H$ by uniform distributions supported by a given, finite number of points. We determine the asymptotics (up to multiplicative constants) of the error of the best approximation for the one-dimensional standard normal distribution, for Gaussian measures as above, and for scalar autonomous SDEs.
\end{abstract}

\section{INTRODUCTION}

We study the approximation of expectations $\mathrm{E}(f(X))$, where $X$ is a random element that takes values in a separable Hilbert space $H$ and where $f: H \rightarrow \mathbb{R}$ is Lipschitz continuous. We consider randomized (Monte Carlo) algorithms that are only allowed to use random bits instead of random numbers. By assumption, all other operations (arithmetic operations, evaluations of elementary functions, and oracle calls to evaluate $f$ ) are performed exactly. Algorithms of this type are called restricted Monte Carlo algorithms, and the approximation of expectations by algorithms of this type will be called random bit quadrature.

Let $\mu$ denote the distribution of $X$. We consider the worst case setting, where randomized algorithms $A$ are compared according to their maximal error $e(A, F, \mu)$ and their maximal cost $\operatorname{cost}(A, F)$ on a class $F$ of functionals $f$. For an arbitrary Monte Carlo algorithm or a restricted Monte Carlo algorithm $\operatorname{cost}(A, F)$ takes into account, in particular, the number of calls of the generator for random numbers or random bits, respectively.

A basic question is to what extent restricted Monte Carlo algorithms are inferior to arbitrary Monte Carlo algorithms. To answer this question one has to compare the $n$-th minimal error

$$
e_{n}^{\text {res }}(F, \mu)=\inf \{e(A, F, \mu): A \text { restricted Monte Carlo algorithm, } \operatorname{cost}(A, F) \leq n\}
$$

2010 Mathematics Subject Classification. 60G15, 60H35, 60H10, 65D30, $65 \mathrm{C} 05$.

Key words and phrases. Gaussian measures on Hilbert spaces, integration, approximation of probability measures, quantization, random bits, multilevel Monte Carlo algorithms, stochastic differential equations. 
of restricted Monte Carlo algorithms with the corresponding quantity $e_{n}(F, \mu)$ for arbitrary Monte Carlo algorithms on classes $F$ of functionals $f$. In the case of infinitedimensional spaces $H$, this question is closely related to three variants of approximation problems for probability measures, namely, quantization, average Kolmogorov widths, and random bit approximation.

In most of the papers on randomized algorithms for continuous problems, uniformly distributed random numbers from $[0,1]$ are assumed to be available. Restricted Monte Carlo algorithms are studied for the classical quadrature problem, where $\mu$ is the uniform distribution on $[0,1]^{d} \subseteq H=\mathbb{R}^{d}$, in, e.g., [11, 14, 22, 23, 24, 29, 31].

In the present paper, we are interested in zero mean Gaussian random elements $X$ with values in a separable Hilbert space $H$ and with a distribution $\mu$ with infinite-dimensional support, and in the class $F=\operatorname{Lip}_{1}$ of all Lipschitz continuous functionals $f: H \rightarrow \mathbb{R}$ with Lipschitz constant at most one.

The Karhunen-Loève expansion of $X$ may be written as

$$
X=\sum_{i=1}^{\infty} \lambda_{i}^{1 / 2} \cdot Y_{i} \cdot e_{i}
$$

with convergence, e.g., in mean-square with respect to the norm of $H$. Here $e_{1}, e_{2}, \ldots$ form an orthonormal system in $H, \lambda_{1} \geq \lambda_{2} \ldots>0$ with $\sum_{i=1}^{\infty} \lambda_{i}<\infty$, and $Y_{1}, Y_{2}, \ldots$ are independent and standard normally distributed random variables. We assume that

$$
\left.\lim _{i \rightarrow \infty} \lambda_{i} \cdot i^{\beta} \cdot(\ln (i))^{\alpha} \in\right] 0, \infty[,
$$

where $\beta>1$ and $\alpha \in \mathbb{R}$. The asymptotic behavior of the variances $\lambda_{i}$ of the random coefficients of $X$ is known in many cases, see, e.g., [18]. For instance,

$$
\beta=2 h+1
$$

and

$$
\alpha=-(d-1) \cdot \beta
$$

for a fractional Brownian sheet $X$ with Hurst parameter $h \in] 0,1\left[\right.$ and $H=L_{2}\left([0,1]^{d}\right)$. In particular, $\beta=2$ and $\alpha=0$ for a Brownian motion, as well as for a Brownian bridge.

For functions $f, g: M \rightarrow[0, \infty]$ on any set $M$ we write $f(m) \preceq g(m)$ if there exists a constant $c>0$ such that $f(m) \leq c \cdot g(m)$ for every $m \in M$. Moreover, $f(m) \succeq g(m)$ means $g(m) \preceq f(m)$ and $f(m) \asymp g(m)$ means $f(m) \preceq g(m)$ and $g(m) \preceq f(m)$. In order to mention the set $M$ explicitly, we sometimes say that the corresponding relation holds uniformly in $m \in M$.

We show that suitable random bit multilevel Monte Carlo algorithms yield the upper bound

$$
e_{n}^{\mathrm{res}}\left(\operatorname{Lip}_{1}, \mu\right) \preceq n^{-\min (1 / 2,(\beta-1) / 2)} \cdot \begin{cases}1, & \text { if } \beta>2, \\ (\ln (n))^{\max (0,1-\alpha / 2)}, & \text { if } \beta=2 \wedge \alpha \neq 2, \\ \ln (\ln (n)), & \text { if } \beta=2 \wedge \alpha=2, \\ (\ln (n))^{-\alpha / 2}, & \text { if } \beta<2,\end{cases}
$$

for the $n$-th minimal error of restricted Monte Carlo algorithms, see Theorem 5 and Corollary 1. See [12] for a recent survey of multilevel algorithms.

Upper and lower bounds for the $n$-th minimal error of arbitrary Monte Carlo algorithms have been established in [3] in a Banach space setting. Combining the upper bound from 
the present paper with the lower bound from [3, Thm. 10] we obtain the following results, see Corollary 1, For $\beta<2$

$$
e_{n}^{\text {res }}\left(\operatorname{Lip}_{1}, \mu\right) \asymp e_{n}\left(\operatorname{Lip}_{1}, \mu\right) \asymp n^{-(\beta-1) / 2} \cdot(\ln (n))^{-\alpha / 2}
$$

and, in particular, there is no superiority of Monte Carlo algorithms over restricted Monte Carlo algorithms in this case. For $\beta=2$, a superiority may at most be present on the level of logarithmic factors, since

$$
\frac{e_{n}^{\mathrm{res}}\left(\operatorname{Lip}_{1}, \mu\right)}{e_{n}\left(\operatorname{Lip}_{1}, \mu\right)} \preceq \begin{cases}\ln (n)^{\max (1, \alpha / 2)}, & \text { if } \alpha \neq 2, \\ \ln (n) \cdot \ln (\ln (n)), & \text { if } \alpha=2 .\end{cases}
$$

For $\beta>2$ we may only conclude that

$$
\liminf _{n \rightarrow \infty} \frac{e_{n}^{\mathrm{res}}\left(\operatorname{Lip}_{1}, \mu\right)}{e_{n}\left(\operatorname{Lip}_{1}, \mu\right)} \preceq(\ln (n))^{(1+\beta) / 2} \cdot(\ln (\ln (n)))^{\alpha / 2} .
$$

Note that for many infinite-dimensional quadrature problems the asymptotic behavior of $n$-th minimal errors is only known up to logarithmic factors. Except for the case $\beta=2$ and $\alpha<1$, the upper bound from the present paper slightly improves the respective bound from [3], which holds true in a Banach space setting.

In [14, random bit quadrature with respect to the uniform distribution $\mu$ on $[0,1]^{d}$ and Sobolev and Hölder classes $F$ of functions on $[0,1]^{d}$ are considered. The $n$-th minimal errors of unrestricted and of restricted Monte Carlo algorithms turn out to be of the same order, and a very small number of $O((2+d) \cdot \log n)$ random bits suffice to achieve asymptotic optimality. The proofs of these results are based on a reduction of the quadrature problem to a summation problem and on a discrete variant of Bakhvalov's trick. See [25] for a related approach to integral equations. Anisotropic function classes are considered in [11, 31].

For the Gaussian measures $\mu$ on infinite-dimensional spaces we do not know whether the number of random bits that are needed to achieve the upper bound for $e_{n}^{\text {res }}\left(\operatorname{Lip}_{1}, \mu\right)$ (or asymptotic optimality) is negligible, compared to $n$. In our construction of a multilevel algorithm that yields the upper bound for $e_{n}^{\text {res }}\left(\operatorname{Lip}_{1}, \mu\right)$ the number of random bits is of the order $n$.

The analysis of random bit quadrature problems leads to the following variant of the quantization problem for probability measures, namely, the optimal approximation of probability measures $\mu$ by uniform distributions $\nu$ on $2^{p}$ points. Since $p$ random bits suffice to sample any such $\nu$, we use the term random bit approximation of probability measures to denote this new type of approximation problem. Let $d$ denote the Wasserstein distance of order two on the set $\mathfrak{M}(H)$ of all Borel probability measures on $H$, and let $\mathfrak{U}(H, p) \subseteq \mathfrak{M}(H)$ denote the set of all uniform distributions on $H$ with support of size $2^{p}$. Given $\mu \in \mathfrak{M}(H)$ we study the distance

$$
\operatorname{rbit}(\mu, p)=\inf \{d(\mu, \nu): \nu \in \mathfrak{U}(H, p)\}
$$

between $\mu$ and $\mathfrak{U}(H, p)$. In the one-dimensional case $H=\mathbb{R}$ this approximation problem has recently been introduced and thoroughly studied for Wasserstein distances of any order in [30], and some of the results from [30] are generalized to the Banach space $\mathbb{R}^{d}$, equipped with the maximum norm, for any $d \in \mathbb{N}$ in [2].

Random bit approximation is closely related to quantization, which has been studied intensively for finite-dimensional and for infinite-dimensional Banach spaces $H$. More 
precisely, let $\mathfrak{F}(H, p)$ denote the set of all Borel probability measures on $H$ with support of size at most $2^{p}$. Obviously the quantization number

$$
\text { quant }(\mu, p)=\inf \{d(\mu, \nu): \nu \in \mathfrak{F}(H, p)\}
$$

is a lower bound for $\operatorname{rbit}(\mu, p)$, i.e.,

$$
\operatorname{rbit}(\mu, p) \geq \text { quant }(\mu, p)
$$

for every $\mu \in \mathfrak{M}(H)$ and every $p \in \mathbb{N}$. A partial list of references on quantization of probability measures includes the monograph [13] and the survey [7] as well as [3, 4, 5, 6. 8, 9, 17, 18, 19]. We stress that the strong asymptotics of quant $(\mu, p)$ is studied most of the time in the literature, while we only consider the weak asymptotics of $\operatorname{rbit}(\mu, p)$. Observe that we lose the control about asymptotic constants anyway in the analysis of the random bit quadrature problem.

For the one-dimensional standard normal distribution $\mu$ we derive

$$
\operatorname{rbit}(\mu, p) \asymp 2^{-p / 2} \cdot p^{-1 / 2}
$$

see Theorem 1, while quant $(\mu, p) \asymp 2^{-p}$ according to a known general result for quantization. For the Gaussian measures $\mu$ on Hilbert spaces we have

$$
\operatorname{rbit}(\mu, p) \asymp p^{-(\beta-1) / 2} \cdot(\ln (p))^{-\alpha / 2},
$$

see Theorems 2 and 3. For scalar autonomous SDEs we consider the distribution $\mu$ of the solution on $L_{2}([0,1])$, and under mild assumptions on the drift and diffusion coefficients we have

$$
\operatorname{rbit}(\mu, p) \asymp p^{-1 / 2},
$$

see Theorem 4. In the latter two cases we only have to establish the upper bound for $\operatorname{rbit}(\mu, p)$, since the matching lower bound even holds for quant $(\mu, p)$, according to known results for quantization.

In the present paper we employ upper bounds for $\operatorname{rbit}(\mu, p)$ and asymptotically optimal random bit approximations to construct random bit algorithms for quadrature and to derive upper bounds for $e^{\text {res }}\left(\operatorname{Lip}_{1}, \mu\right)$. In [3] close relations between quantization numbers and average Kolmogorov widths on the one-hand side, and upper and lower bounds for (minimal) errors of arbitrary Monte Carlo algorithms have been established.

This work is partially motivated by reconfigurable architectures like field programmable gate arrays (FPGAs). These devices allow users to choose the precision of each individual operation on a bit level and provide a generator for random bits. In the setting and analysis of the present paper we take into account the latter fact, while we ignore all finite precision issues for arithmetic operations. We refer to [1, 27] for the construction and for extensive tests of a finite precision multilevel algorithm for FPGAs with applications in computational finance. For an error analysis of the Euler scheme for SDEs in a finite precision arithmetic we refer to [26].

This paper is organized as follows. In Section 2 we formulate and study the random bit approximation problem for probability measures. Section 3 is devoted to the analysis of random bit quadrature with respect to Gaussian measures. In the Appendix we derive some asymptotic properties of the distribution function and its inverse for the standard normal distribution. 


\section{Random Bit Approximation of Probability Measures}

2.1. Definitions and Basic Properties. Consider the set $\mathfrak{M}(V)$ of all Borel probability measures on a separable Banach space $\left(V,\|\cdot\|_{V}\right)$ with a finite second moment, equipped with the Wasserstein distance $d$ of order two, i.e.,

$$
d\left(\mu_{1}, \mu_{2}\right)=\inf \left\{\left(\mathrm{E}\left\|X_{1}-X_{2}\right\|_{V}^{2}\right)^{1 / 2}: P_{X_{1}}=\mu_{1}, P_{X_{2}}=\mu_{2}\right\}
$$

for $\mu_{1}, \mu_{2} \in \mathfrak{M}(V)$. Here $X_{1}$ and $X_{2}$ are jointly defined on any probability space and take values in $V$, and $P_{X_{i}}$ denotes the distribution of $X_{i}$.

For $p \in \mathbb{N}$ we use $\nu^{(p)}$ to denote the uniform distribution on $\{0,1\}^{p}$, and we define

$$
\mathfrak{R}(V, p)=\left\{\nu_{f}^{(p)} \in \mathfrak{M}(V): f:\{0,1\}^{p} \rightarrow V\right\},
$$

where $\nu_{f}^{(p)}$ denotes the distribution of $f$ with respect to $\nu^{(p)}$. Observe that $\mathfrak{R}(V, p)$ is the set of all probability measures on $V$ with support of size at most $2^{p}$ and with probability weights being integer multiples of $1 / 2^{p}$. Clearly $p$ random bits suffice to sample from any $\nu \in \mathfrak{R}(V, p)$.

Given $\mu \in \mathfrak{M}(V)$ we study the distance

$$
\operatorname{rbit}(\mu, p)=\inf \{d(\mu, \nu): \nu \in \mathfrak{R}(V, p)\}
$$

between $\mu$ and $\mathfrak{R}(V, p)$. We wish to determine the asymptotic behavior of $\operatorname{rbit}(\mu, p)$ as $p$ tends to infinity and to construct probability measures $\mu^{(p)} \in \mathfrak{R}(V, p)$ such that $d\left(\mu, \mu^{(p)}\right)$ is close to $\operatorname{rbit}(\mu, p)$.

Specifically, we are interested in separable Hilbert spaces $\left(H,\|\cdot\|_{H}\right)$ and the cases of $\mu$ being the one-dimensional standard normal distribution, the distribution of a Brownian bridge on $H=L_{2}([0,1])$ or, more generally, of a Gaussian random element on an infinitedimensional Hilbert space $H$, and finally the distribution of the solution of a scalar SDE on $H=L_{2}([0,1])$.

Remark 1. Obviously,

$$
\operatorname{rbit}(\mu, p+1) \leq \operatorname{rbit}(\mu, p)
$$

for every $\mu \in \mathfrak{M}(V)$ and every $p \in \mathbb{N}$.

Remark 2. Let

$$
\mathfrak{U}(V, p)=\left\{\nu_{f}^{(p)} \in \mathfrak{M}(V): f:\{0,1\}^{p} \rightarrow V \text { is injective }\right\},
$$

which is the set of all uniform distributions on $V$ with support of size $2^{p}$. Since $\mathfrak{U}(V, p) \subseteq$ $\mathfrak{R}(V, p)$ with a dense embedding with respect to the Wasserstein distance $d$, we have

$$
\operatorname{rbit}(\mu, p)=\inf \{d(\mu, \nu): \nu \in \mathfrak{U}(V, p)\}
$$

for every $\mu \in \mathfrak{M}(V)$ and every $p \in \mathbb{N}$. Consequently, random bit approximation deals with the optimal approximation of probability measures by uniform distributions on $2^{p}$ points.

The one-dimensional case $V=\mathbb{R}$ has been thoroughly studied in a more general setting in [30], and some of the results in the latter paper have been generalized in [2] to the Banach space $V=\mathbb{R}^{d}$, equipped with the maximum norm, for any $d \in \mathbb{N}$. Given $p \in \mathbb{N}$ and probability weights $a_{1}, \ldots, a_{2^{p}}$ the objective is to minimize the Wasserstein distance of order $r$ between a Borel probability measure $\mu$ on $V$ with a finite moment of order $r$ and $\nu=\sum_{k=1}^{2^{p}} a_{k} \cdot \delta_{x_{k}}$ with Dirac measures $\delta_{x_{k}}$ at any points $x_{k}$. This problem is called best finite constrained approximation with prescribed weights $a_{k}$ in [30]. 
The special case $V=\mathbb{R}, r=2$, and $a_{k}=2^{-p}$ corresponds to the random bit approximation of $\mu \in \mathfrak{M}(\mathbb{R})$, and we present key results from [30] in this case. In the sequel, $\Psi^{-1}$ denotes the inverse of the distribution function of $\mu$.

Remark 3. According to [30, Rem. 5.6(ii)], the unique best approximation $\nu \in \mathfrak{R}(\mathbb{R}, p)$ of $\mu \in \mathfrak{M}(\mathbb{R})$ with respect to $d$ is determined by the points

$$
x_{k}^{*}=2^{p} \cdot \int_{(k-1) \cdot 2^{-p}}^{k \cdot 2^{-p}} \Psi^{-1}(t) \mathrm{d} t, \quad k=1, \ldots, 2^{p} .
$$

Assume that the measure corresponding to $\Psi^{-1}$ is absolutely continuous with respect to the Lebesgue measure on $[0,1]$. In [30, Thm 5.15] a constant $c \in] 0, \infty[\cup\{\infty\}$ is given explicitly such that

$$
\lim _{p \rightarrow \infty} 2^{p} \cdot \operatorname{rbit}(\mu, p)=c .
$$

In particular, $\operatorname{rbit}(\mu, p) \succeq 2^{-p}$, and this lower bound is sharp if and only if $c<\infty$. As an elementary example we have $c=(2 \cdot \sqrt{3})^{-1}$ for $\mu$ being the uniform distribution on the unit interval.

Next, assume that all moments of $\mu$ are finite. Then we have

$$
\operatorname{rbit}(\mu, p) \preceq\left(2^{p}\right)^{-1 / 2+\varepsilon}
$$

for all $\varepsilon>0$, see [30, Thm. 5.20].

Remark 4. Random bit approximation is closely related to quantization, which has been studied intensively for finite-dimensional and for infinite-dimensional spaces $V$. More precisely, let

$$
\mathfrak{F}(V, p)=\left\{\nu \in \mathfrak{M}(V):|\operatorname{supp}(\nu)| \leq 2^{p}\right\}
$$

denote the set of all probability measures on $V$ with support of size at most $2^{p}$. The quantization numbers

$$
\text { quant }(\mu, p)=\inf \{d(\mu, \nu): \nu \in \mathfrak{F}(V, p)\}
$$

immediately yield lower bounds for $\operatorname{rbit}(\mu, p)$, i.e.,

$$
\operatorname{rbit}(\mu, p) \geq \text { quant }(\mu, p)
$$

for every $\mu \in \mathfrak{M}(V)$ and every $p \in \mathbb{N}$. A partial list of references on quantization of probability measures includes the monograph [13] and the survey [7] as well as [3, 4, [5, 6, 8, 9, 17, 18, 19].

The results from [10], which deals with quantization on $V=\mathbb{R}^{d}$ by means of empirical measures, immediately yield upper bounds for $\operatorname{rbit}(\mu, p)$. In particular, if $d \geq 5$ and if $\mu$ has a finite moment of any order greater than $2 /(1-2 / d)$, then $\operatorname{rbit}(\mu, p) \preceq 2^{-p / d}$, see [10, Thm. 1]. This upper bound is sharp in many cases, since quant $(\mu, p) \succeq 2^{-p / d}$ under mild assumptions on $\mu$ for every $d \in \mathbb{N}$, see [7, 13] for details.

We stress that the strong asymptotics of quant $(\mu, p)$ is studied most of the time in the literature, while we only consider the weak asymptotics of $\operatorname{rbit}(\mu, p)$. Observe that we lose the control about asymptotic constants anyway in the analysis of the random bit quadrature problem.

Remark 5. Let $\left(W,\|\cdot\|_{W}\right)$ denote another separable Banach space. For the proof of upper bounds for $\operatorname{rbit}(\mu, p)$ we may use the following simple observation. Let $f: W \rightarrow V$ be measurable and $\mu \in \mathfrak{R}(W, p)$, then $\mu_{f} \in \mathfrak{R}(V, p)$. 
2.2. Approximation of the Standard Normal Distribution. We first fix some notations. For $p \in \mathbb{N}$ let

$$
\begin{aligned}
D^{(p)} & =\left\{\sum_{i=1}^{p} b_{i} \cdot 2^{-i}+2^{-(p+1)}: b_{i} \in\{0,1\} \text { for } i=1, \ldots, p\right\} \\
& =\left\{k \cdot 2^{-p}-2^{-(p+1)}: k=1, \ldots, 2^{p}\right\}
\end{aligned}
$$

denote the set of dyadic numbers from $\left[0,1\right.$ [ with $p$ bits, shifted by $2^{-(p+1)}$, so that $D^{(p)}$ is symmetric with respect to $1 / 2$. Furthermore, we define the truncation operator $T^{(p)}$ via

$$
T^{(p)}:\left[0,1\left[\rightarrow D^{(p)}, x \mapsto \frac{\left\lfloor 2^{p} x\right\rfloor}{2^{p}}+2^{-(p+1)},\right.\right.
$$

i.e., the application of $T^{(p)}$ means rounding to a nearest element from $D^{(p)}$.

Let $Y$ be a standard normally distributed random variable and let $\Phi$ denote the corresponding distribution function. Observe that $U=\Phi(Y)$ is uniformly distributed on $[0,1]$, so that $T^{(p)}(U)$ is uniformly distributed on $D^{(p)}$. The distribution of

$$
Y^{(p)}=\Phi^{-1} \circ T^{(p)} \circ \Phi(Y)
$$

therefore belongs to $\mathfrak{U}(\mathbb{R}, p)$.

Theorem 1. Let $\mu$ denote the standard normal distribution. Then we have

$$
\operatorname{rbit}(\mu, p) \asymp 2^{-p / 2} \cdot p^{-1 / 2} .
$$

Furthermore, let $Y^{(p)}$ as in (44). Then

$$
\left(\mathrm{E}\left|Y-Y^{(p)}\right|^{2}\right)^{1 / 2} \asymp \operatorname{rbit}(\mu, p) .
$$

Moreover,

$$
\mathrm{E}\left(Y^{(p)}\right)=0
$$

and

$$
\sup _{p \in \mathbb{N}} \mathrm{E}\left|Y^{(p)}\right|^{r}<\infty
$$

for all $r \geq 1$.

Proof. By definition,

$$
\operatorname{rbit}(\mu, p) \leq\left(\mathrm{E}\left|Y-Y^{(p)}\right|^{2}\right)^{1 / 2} .
$$

Hence we show that

$$
\left(\mathrm{E}\left|Y-Y^{(p)}\right|^{2}\right)^{1 / 2} \preceq 2^{-p / 2} \cdot p^{-1 / 2} .
$$

Let $z_{k}=k \cdot 2^{-p}$ for $k=2^{p-1}, \ldots, 2^{p}$, and let $\varphi$ denote the density of the standard normal distribution. We have

$$
\mathrm{E}\left|Y-Y^{(p)}\right|^{2}=\mathrm{E}\left|\Phi^{-1}(U)-\Phi^{-1} \circ T^{(p)}(U)\right|^{2}=2 \cdot \sum_{k=2^{p-1}+1}^{2^{p}} A_{k}
$$

where

$$
A_{k}=\int_{\left[z_{k-1}, z_{k}[\right.}\left|\Phi^{-1}(u)-x_{k}\right|^{2} \mathrm{~d} u
$$

with $x_{k}=\Phi^{-1}\left(z_{k}-2^{-(p+1)}\right)$. 
Consider the case $2^{p-1}+1 \leq k \leq 2^{p}-2$. Observe that $\varphi \circ \Phi^{-1}$ is monotonically decreasing on $[1 / 2,1[$. Using

$$
\left|\Phi^{-1}(u)-x_{k}\right| \leq \frac{\left|u-\left(z_{k}-2^{-(p+1)}\right)\right|}{\varphi\left(\Phi^{-1}\left(z_{k}\right)\right)}
$$

for $u \in\left[z_{k-1}, z_{k}[\right.$, we obtain

$$
A_{k} \leq \frac{2^{-3 p}}{12 \varphi^{2}\left(\Phi^{-1}\left(z_{k}\right)\right)}
$$

Consequently,

$$
\begin{aligned}
2^{p} p \cdot \sum_{k=2^{p-1}+1}^{2^{p}-2} A_{k} & \leq \frac{2^{-2 p} p}{12} \cdot \sum_{k=2^{p-1}+1}^{2^{p}-2} \frac{1}{\varphi^{2}\left(\Phi^{-1}\left(z_{k}\right)\right)} \leq \frac{2^{-p} p}{12} \cdot \int_{1 / 2}^{1-2^{-p}} \frac{1}{\varphi^{2}\left(\Phi^{-1}(u)\right)} \mathrm{d} u \\
& =\frac{2^{-p} p \cdot \sqrt{2 \pi}}{12} \cdot h\left(\Phi^{-1}\left(1-2^{-p}\right)\right)
\end{aligned}
$$

where

$$
h(a)=\int_{0}^{a} \exp \left(x^{2} / 2\right) \mathrm{d} x
$$

for $a>0$. By Lemma 8 from the Appendix we obtain

$$
\limsup _{p \rightarrow \infty} 2^{p} p \cdot \sum_{k=2^{p-1}+1}^{2^{p}-2} A_{k} \leq \frac{1}{12 \ln 4} .
$$

For every $2^{p-1}+1 \leq k \leq 2^{p}$

$$
A_{k} \leq 2 \cdot \int_{\left[z_{k-1}+2^{-(p+1)}, z_{k}[\right.}\left|\Phi^{-1}(u)-x_{k}\right|^{2} \mathrm{~d} u=2 \cdot \int_{\left[x_{k}, \Phi^{-1}\left(z_{k}\right)[\right.}\left(x-x_{k}\right)^{2} \varphi(x) \mathrm{d} x .
$$

Furthermore, this upper bound for $A_{k}$ is monotonically increasing in $k$, since $\Phi^{-1}$ is convex on $\left[1 / 2,1\left[\right.\right.$. Therefore we consider the case $k=2^{p}$. Put

$$
g(a)=\int_{[a, \infty[}(x-a)^{2} \varphi(x) \mathrm{d} x
$$

for $a>0$. By Lemma 9 from the Appendix we obtain

$$
\limsup _{p \rightarrow \infty} 2^{p} p \cdot \mathrm{E}\left|Y-Y^{(p)}\right|^{2} \leq \frac{49}{6 \ln 4},
$$

which completes the proof of the upper bound (10).

Next we show

$$
\operatorname{rbit}(\mu, p) \succeq 2^{-p / 2} \cdot p^{-1 / 2}
$$

To this end we consider a random variable $\widehat{Y}^{(p)}$ with distribution in $\mathfrak{R}(\mathbb{R}, p)$ and defined on the same space as $Y$. Let $\widehat{x}$ denote the essential supremum of $\widehat{Y}^{(p)}$. We are going to consider two cases, at first we assume

$$
\widehat{x} \leq \Phi^{-1}\left(1-2^{-(p+1)}\right) .
$$

Due to the monotonicity of $\Phi^{-1}$ we have

$$
\begin{aligned}
\mathrm{E}\left(Y-\widehat{Y}^{(p)}\right)^{2} & \geq \mathrm{E}\left(\left(\Phi^{-1}(U)-\widehat{Y}^{(p)}\right)^{2} \cdot 1_{\left\{U \geq 1-2^{-(p+1)}\right\}}\right) \\
& \geq \mathrm{E}\left(\left(\Phi^{-1}(U)-\Phi^{-1}\left(1-2^{-(p+1)}\right)\right)^{2} \cdot 1_{\left\{U \geq 1-2^{-(p+1)}\right\}}\right) \\
& =g\left(\Phi^{-1}\left(1-2^{-(p+1)}\right)\right) .
\end{aligned}
$$


Lemma 9 from the Appendix yields $g\left(\Phi^{-1}\left(1-2^{-(p+1)}\right)\right) \succeq 2^{-p} \cdot p^{-1}$. For the second case, i.e.,

$$
\widehat{x}>\Phi^{-1}\left(1-2^{-(p+1)}\right)
$$

we use Lemma 11 from the Appendix with $a=1-3 \cdot 2^{-(p+2)}$ and $b=1-2^{-(p+1)}$ to conclude that

$$
\Phi^{-1}(b)-\Phi^{-1}(a) \succeq p^{-1 / 2} .
$$

Together with the monotonicity of $\Phi^{-1}$ this leads to

$$
\begin{aligned}
\mathrm{E}\left(Y-\widehat{Y}^{(p)}\right)^{2} & \geq \mathrm{E}\left(\left(\Phi^{-1}(U)-\widehat{x}\right)^{2} \cdot 1_{\left\{\widehat{Y}^{(p)}=\widehat{x}\right\}} \cdot 1_{\{U \leq a\}}\right) \\
& \geq \mathrm{E}\left(\left(\Phi^{-1}(a)-\Phi^{-1}(b)\right)^{2} \cdot 1_{\left\{\widehat{Y}^{(p)}=\widehat{x}\right\}} \cdot 1_{\{U \leq a\}}\right) \\
& \succeq p^{-1} \cdot P\left(\left\{\widehat{Y}^{(p)}=\widehat{x}\right\} \cap\{U \leq a\}\right) \\
& \geq p^{-1} \cdot\left(P\left(\left\{\widehat{Y}^{(p)}=\widehat{x}\right\}\right)-P(\{U>a\})\right) \\
& \geq p^{-1} \cdot\left(2^{-p}-P(\{U>a\})\right) \\
& =p^{-1} \cdot 2^{-(p+2)} .
\end{aligned}
$$

This completes the proof of (13). Combing (9), (10), and (13) yields (5) and (6).

For the proof of (7) we observe that $T^{(p)}(U)$ is uniformly distributed on $D^{(p)}$. Since $\Phi^{-1}(1-x)=-\Phi^{-1}(x)$ for $\left.x \in\right] 0,1[$, we get (17) for symmetry reasons.

Observe that $x \mapsto\left(\Phi^{-1}(x)\right)^{r}$ is a convex function on $[1 / 2,1$ [ for $r \geq 1$ with integral equal to $\frac{1}{2} \mathrm{E}|Y|^{r}$, and applying a midpoint rule to this function we get $\frac{1}{2} \mathrm{E}\left|Y^{(p)}\right|^{r}$. Hence we have

$$
\sup _{p \in \mathbb{N}} \mathrm{E}\left|Y^{(p)}\right|^{r} \leq \mathrm{E}|Y|^{r}<\infty
$$

which implies (8).

Remark 6. We compare Theorem 1 with the results from [30], as discussed in Remark 3, for the standard normal distribution $\mu$.

Note that the measure corresponding to $\Phi^{-1}$ is absolutely continuous with respect to the Lebesgue measure on $[0,1]$. Theorem 1 implies that $c=\infty$ in (2). Moreover, the order of convergence of $\operatorname{rbit}(\mu, p)$ is only slightly better than the upper bound (3) , which holds for every $\mu \in \mathfrak{M}(\mathbb{R})$ with finite moments of any order.

The optimal selection of support points $x_{k}^{*}$ is given by the local averages of $\Phi^{-1}$ based on a uniform partition of $[0,1]$, see (11) with $\Psi^{-1}=\Phi^{-1}$. In Theorem 1 we consider a slightly simpler construction, which still yields the same order of convergence of the Wasserstein distance, as we employ the values $x_{k}$ of $\Phi^{-1}$ at the midpoints for this partition. Both of these point sets are symmetric with respect to $1 / 2$, and $x_{k}<x_{k}^{*}<x_{k+1}$ for $k=2^{p-1}+1, \ldots, 2^{p}-1$.

Remark 7. We compare Theorem 1 with known results for the quantization problem, see, e.g., [13, Thm. 6.2]. First of all,

$$
\text { quant }(\mu, p) \asymp 2^{-p}
$$


for the quantization of the standard normal distribution $\mu$, so that the quantization error converges to zero much faster than the corresponding quantity for random bit approximation. On the other hand, for the uniform distribution on $[0,1]$ both quantities are of the same order $2^{-p}$.

2.3. Approximation of the Distribution of a Brownian Bridge. Let $\left(s_{i}\right)_{i \in \mathbb{N}}$ be the sequence of Schauder functions given by

$$
s_{i}(t)=\int_{0}^{t} h_{i}(u) \mathrm{d} u, \quad t \in[0,1]
$$

with

$$
h_{2^{m}+k-1}=2^{m / 2} \cdot\left(1_{I_{2^{m}+k}}-1_{J_{2^{m}+k}}\right)
$$

for $m \in \mathbb{N}_{0}$ and $k=1, \ldots, 2^{m}$, where

$$
I_{2^{m}+k}=\left[(k-1) / 2^{m},(k-1 / 2) / 2^{m}[\right.
$$

and

$$
J_{2^{m}+k}=\left[(k-1 / 2) / 2^{m}, k / 2^{m}[.\right.
$$

Let $B$ denote a standard Brownian bridge on $[0,1]$, which is henceforth considered as a centered Gaussian random element that takes values in $H=L_{2}=L_{2}([0,1])$. The Lévy-Ciesielski (or Brownian bridge) representation of $B$ states that

$$
B=\sum_{i=1}^{\infty} Y_{i} \cdot s_{i}
$$

with convergence, e.g., in mean-square with respect to the $L_{2}$-norm. Here $Y_{1}, Y_{2}, \ldots$ is an independent sequence of standard normally distributed random variables.

We define

$$
B^{(\ell)}=\sum_{i=1}^{2^{\ell}-1} Y_{i} \cdot s_{i}
$$

for $\ell \in \mathbb{N}$, i.e., $B^{(\ell)}$ is the piecewise linear interpolation of $B$ at the points $k \cdot 2^{-\ell}$ with $k=0, \ldots, 2^{\ell}$. The following result is well known, see, e.g., [28, Sec. II.3] for references and remarks.

Lemma 1. We have

$$
\left(\mathrm{E}\left\|B-B^{(\ell)}\right\|_{L_{2}}^{2}\right)^{1 / 2} \asymp 2^{-\ell / 2} .
$$

For $\ell \in \mathbb{N}$ we consider a vector

$$
\boldsymbol{p}=\left(p_{1}, \ldots, p_{2^{\ell}-1}\right) \in \mathbb{N}^{2^{\ell}-1}
$$

of bit numbers. We define

$$
B^{(\ell, \boldsymbol{p})}=\sum_{i=1}^{2^{\ell}-1} Y_{i}^{\left(p_{i}\right)} \cdot s_{i},
$$

where $Y_{i}^{\left(p_{i}\right)}$ is the approximation of $Y_{i}$ according to (4). This approach, which is appropriate for the construction of multilevel algorithms, see Section 3, has been suggested in [12, p. 320]. Note that the distribution of $B^{(\ell, \boldsymbol{p})}$ belongs to $\mathfrak{U}\left(L_{2},|\boldsymbol{p}|\right)$ with

$$
|\boldsymbol{p}|=\sum_{i=1}^{2^{\ell}-1} p_{i}
$$


Lemma 2. We have

$$
\left(\mathrm{E}\left\|B^{(\ell)}-B^{(\ell, \boldsymbol{p})}\right\|_{L_{2}}^{2}\right)^{1 / 2} \asymp\left(\sum_{i=1}^{2^{\ell}-1} 2^{-p_{i}} / p_{i} \cdot i^{-2}\right)^{1 / 2}
$$

uniformly in $\ell \in \mathbb{N}$ and $\boldsymbol{p} \in \mathbb{N}^{2^{\ell}-1}$.

Proof. Let $\widehat{Y}_{i}=Y_{i}^{\left(p_{i}\right)}$. Use Theorem 1 and $\left\|s_{i}\right\|_{L_{2}}^{2} \asymp i^{-2}$ to obtain

$$
\begin{aligned}
\mathrm{E}\left\|B^{(\ell, \boldsymbol{p})}-B^{(\ell)}\right\|_{L_{2}}^{2}=\int_{0}^{1} \mathrm{E}\left(\sum_{i=1}^{2^{\ell}-1}\left(\widehat{Y}_{i}-Y_{i}\right) \cdot s_{i}(t)\right)^{2} \mathrm{~d} t \\
=\int_{0}^{1} \operatorname{Var}\left(\sum_{i=1}^{2^{\ell}-1}\left(\widehat{Y}_{i}-Y_{i}\right) \cdot s_{i}(t)\right) \mathrm{d} t=\int_{0}^{1} \sum_{i=1}^{2^{\ell}-1} \operatorname{Var}\left(\left(\widehat{Y}_{i}-Y_{i}\right) \cdot s_{i}(t)\right) \mathrm{d} t \\
=\sum_{i=1}^{2^{\ell}-1} \mathrm{E}\left(\widehat{Y}_{i}-Y_{i}\right)^{2} \cdot \int_{0}^{1} s_{i}^{2}(t) \mathrm{d} t \asymp \sum_{i=1}^{2^{\ell}-1} 2^{-p_{i}} / p_{i} \cdot i^{-2} .
\end{aligned}
$$

Theorem 2. Let $\mu$ be the distribution of a standard Brownian bridge $B$ on $L_{2}$. Then we have

$$
\operatorname{rbit}(\mu, p) \asymp \operatorname{quant}(\mu, p) \asymp p^{-1 / 2} .
$$

Define $\boldsymbol{p}(\ell) \in \mathbb{N}^{2^{\ell}-1}$ for $\ell \in \mathbb{N}$ by

$$
p_{i}(\ell)=2 \cdot\left(\ell-\left\lfloor\log _{2} i\right\rfloor\right), \quad i=1, \ldots, 2^{\ell}-1 .
$$

Then we have

$$
\left(\mathrm{E}\left\|B-B^{(\ell, \boldsymbol{p}(\ell))}\right\|_{L_{2}}^{2}\right)^{1 / 2} \asymp \operatorname{rbit}(\mu,|\boldsymbol{p}(\ell)|)
$$

and

$$
|\boldsymbol{p}(\ell)|=2^{\ell+2}-2 \ell-4 \asymp 2^{\ell} .
$$

Proof. We write $\boldsymbol{p}$ and $p_{i}$ instead of $\boldsymbol{p}(\ell)$ and $p_{i}(\ell)$, respectively, to simplify the notation. By definition,

Hence we show that

$$
\operatorname{rbit}(\mu,|\boldsymbol{p}|) \leq\left(\mathrm{E}\left\|B-B^{(\ell, \boldsymbol{p})}\right\|_{L_{2}}^{2}\right)^{1 / 2}
$$

$$
\left(\mathrm{E}\left\|B-B^{(\ell, \boldsymbol{p})}\right\|_{L_{2}}^{2}\right)^{1 / 2} \preceq|\boldsymbol{p}|^{-1 / 2}
$$

Since

$$
\sum_{i=1}^{2^{\ell}-1} 2^{-p_{i}} / p_{i} \cdot i^{-2} \leq 2^{-\ell}
$$

for the specific choice of the bit numbers $p_{i}$, Lemmata 1 and 2 yield

$$
\left(\mathrm{E}\left\|B-B^{(\ell, p)}\right\|_{L_{2}}^{2}\right)^{1 / 2} \preceq 2^{-\ell / 2} .
$$

The explicit formula for $|\boldsymbol{p}|$ is easily verified by induction, and this completes the proof of the asymptotic upper bound (15). On the other hand,

$$
\text { quant }(\mu, p) \asymp p^{-1 / 2} \text {, }
$$

see [17, p. 527] and [4]. 
Remark 8. Observe that the Schauder function $s_{i}$ has a support of size $2^{-\left\lfloor\log _{2} i\right\rfloor}$. Therefore $B^{(\ell, \boldsymbol{p}(\ell))}$ involves all Schauder functions with support size between 1 and $2^{-(\ell-1)}$, and the number of random bits that is associated to $s_{i}$ according to (14) only depends on the size of its support. This number varies linearly between $2 \ell$ for $s_{1}$ and 2 for $s_{i}$ with $i=2^{\ell-1}, \ldots, 2^{\ell}-1$.

In our construction the total number $|\boldsymbol{p}(\ell)|$ of bits coincides, up to a multiplicative constant, with the numbers of terms in $B^{(\ell, p(\ell))}$ and in $B^{(\ell)}$. The partial sum $B^{(\ell)}$ formally corresponds to $p_{i}(\ell)=\infty$ for $i=1, \ldots, 2^{\ell}-1$, but still the errors of $B^{(\ell)}$ and $B^{\ell, \boldsymbol{p}(\ell))}$ are of the same order $2^{-\ell / 2}$, see Lemma 1 and Theorem 2 .

Remark 9. The bit numbers given by (14) depend on $i$ and they approximately minimize $|\boldsymbol{p}|$, subject to the constraint (16).

For constant bit numbers

$$
p=p_{1}=\ldots=p_{2^{\ell}-1}
$$

the following holds true. For (16) to hold true we must have $2^{-p} / p \leq 2^{-\ell}$, and together with $p \leq 2^{p}$ this yields $p \geq \ell / 2$. On the other hand $p=2 \cdot \ell$ implies (16). Therefore the minimum of $|\boldsymbol{p}|$, subject to the constraints (16) and (17) is only of the order $2^{\ell} \cdot \ell$.

2.4. Approximation of Gaussian Measures. In this section we consider a centered Gaussian random element $X$ that takes values in a separable Hilbert space $\left(H,\|\cdot\|_{H}\right)$ and has an infinite-dimensional support. The Karhunen-Loève expansion of $X$ may be written as

$$
X=\sum_{i=1}^{\infty} \lambda_{i}^{1 / 2} \cdot Y_{i} \cdot e_{i}
$$

with convergence, e.g., in mean-square with respect to the norm of $H$. Here $\left(e_{i}\right)_{i \in \mathbb{N}}$ is an orthonormal system in $H$ and $\left(\lambda_{i}\right)_{i \in \mathbb{N}}$ is a non-increasing and summable sequence of strictly positive numbers, and $Y_{1}, Y_{2}, \ldots$ is an independent sequence of standard normally distributed random variables. We assume that

$$
\left.\lim _{i \rightarrow \infty} \lambda_{i} \cdot i^{\beta} \cdot(\ln (i))^{\alpha} \in\right] 0, \infty[,
$$

where $\beta>1$ and $\alpha \in \mathbb{R}$. The asymptotic behavior of the variances $\lambda_{i}$ of the random coefficients of $X$ is known in many cases. For instance,

$$
\beta=2 h+1
$$

and

$$
\alpha=-(d-1) \cdot \beta
$$

for a fractional Brownian sheet $X$ on $[0,1]^{d}$ with Hurst parameter $\left.h \in\right] 0,1[$ and $H=$ $L_{2}\left([0,1]^{d}\right)$, see, e.g., [18, p. 1586, p. 1588]. In particular, $\beta=2$ and $\alpha=0$ for a Brownian motion, as well as for a Brownian bridge.

The analysis from the previous section extends to the case of Gaussian random elements in a straight-forward way. In contrast to the Lévy-Ciesielski representation the KarhunenLoève expansion may naturally be truncated after any number of terms. For $m \in \mathbb{N}$ we consider a vector

$$
\boldsymbol{p}=\left(p_{1}, \ldots, p_{m}\right) \in \mathbb{N}^{m}
$$


of bit numbers. We define

$$
X^{(m, p)}=\sum_{i=1}^{m} \lambda_{i}^{1 / 2} \cdot Y_{i}^{\left(p_{i}\right)} \cdot e_{i},
$$

where $Y_{i}^{\left(p_{i}\right)}$ is the approximation of $Y_{i}$ according to (44). Note that the distribution of $X^{(m, p)}$ belongs to $\mathfrak{U}(H,|\boldsymbol{p}|)$ with

$$
|\boldsymbol{p}|=\sum_{i=1}^{m} p_{i} .
$$

Theorem 3. Let $\mu$ denote the distribution of the Gaussian random element $X$ on $H$, and assume that (19) is satisfied. Then we have

$$
\operatorname{rbit}(\mu, p) \asymp \operatorname{quant}(\mu, p) \asymp p^{-(\beta-1) / 2} \cdot(\ln (p+1))^{-\alpha / 2} .
$$

Define $\boldsymbol{p}(m) \in \mathbb{N}^{m}$ for $m \in \mathbb{N}$ by

$$
p_{i}(m)=\left\lceil\max \left(\tilde{p}_{i}(m), 1\right)\right\rceil, \quad i=1, \ldots, m,
$$

where

$$
\tilde{p}_{i}(m)=\beta \cdot \log _{2}(m / i)+\max (\alpha, 0) \cdot \log _{2}\left(\log _{2}(m+1) / \log _{2}(i+1)\right) .
$$

Then we have

$$
\left(\mathrm{E}\left\|X-X^{(m, p(m))}\right\|_{H}^{2}\right)^{1 / 2} \asymp \operatorname{rbit}(\mu,|\boldsymbol{p}(m)|)
$$

and

$$
|\boldsymbol{p}(m)| \asymp m \text {. }
$$

Proof. We write $\boldsymbol{p}, p_{i}$, and $\tilde{p}_{i}$ instead of $\boldsymbol{p}(m), p_{i}(m)$, and $\tilde{p}_{i}(m)$, respectively, to simplify the notation. Note that

$$
\int_{0}^{1} \frac{1}{x \cdot t+1} d t=\frac{\ln (x+1)}{x}
$$

for $x>0$. It follows that $x \mapsto x / \log _{2}(x+1)$ is strictly increasing on $] 0, \infty[$. Therefore $m / i \geq \log _{2}(m+1) / \log _{2}(i+1)$ and

$$
p_{i} \leq 1+\tilde{p}_{i} \preceq 1+\ln (m / i)
$$

uniformly in $m \in \mathbb{N}$ and $i=1, \ldots, m$. Since

$$
\sum_{i=2}^{m} \ln (m / i) \leq \int_{1}^{m} \ln (m / x) \mathrm{d} x=m-\ln (m)-1,
$$

we obtain

$$
|\boldsymbol{p}| \preceq m+\sum_{i=1}^{m} \ln (m / i) \leq 2 m-1,
$$

while $|\boldsymbol{p}| \geq m$ trivially holds true. We conclude that $|\boldsymbol{p}| \asymp m$, as claimed.

By definition,

Hence we show that

$$
\operatorname{rbit}(\mu,|\boldsymbol{p}|) \leq\left(\mathrm{E}\left\|X-X^{(m, p)}\right\|_{H}^{2}\right)^{1 / 2}
$$

$$
\left(\mathrm{E}\left\|X-X^{(m, p)}\right\|_{H}^{2}\right)^{1 / 2} \preceq m^{-(\beta-1) / 2} \cdot(\ln (m+1))^{-\alpha / 2} .
$$

First of all,

$$
X^{(m)}=\sum_{i=1}^{m} \lambda_{i}^{1 / 2} \cdot Y_{i} \cdot e_{i}
$$


with $m \in \mathbb{N}$ satisfies

$$
\mathrm{E}\left\|X-X^{(m)}\right\|_{H}^{2} \asymp m^{-(\beta-1)} \cdot(\ln (m+1))^{-\alpha},
$$

see (19). Furthermore, Theorem 1 yields

$$
\mathrm{E}\left\|X^{(m)}-X^{(m, \boldsymbol{p})}\right\|_{H}^{2}=\sum_{i=1}^{m} \mathrm{E}\left(Y_{i}-Y_{i}^{\left(p_{i}\right)}\right)^{2} \cdot \lambda_{i} \asymp \sum_{i=1}^{m} 2^{-p_{i}} / p_{i} \cdot i^{-\beta} \cdot(\ln (i+1))^{-\alpha}
$$

uniformly in $m \in \mathbb{N}$ and $\boldsymbol{p} \in \mathbb{N}^{m}$. For the specific choice of bit numbers $p_{i}$ we obtain

$$
2^{-p_{i}} \cdot i^{-\beta} \cdot(\ln (i+1))^{-\alpha} \preceq m^{-\beta} \cdot(\ln (m+1))^{-\alpha}
$$

uniformly in $m \in \mathbb{N}$ and $i=1, \ldots, m$. Since $p_{i} \geq 1$, we conclude that

$$
\mathrm{E}\left\|X^{(m)}-X^{(m, \boldsymbol{p})}\right\|_{H}^{2} \preceq m^{-(\beta-1)} \cdot(\ln (m+1))^{-\alpha},
$$

which completes the proof of (21).

On the other hand,

$$
\text { quant }(\mu, p) \asymp p^{-(\beta-1) / 2} \cdot(\ln (p+1))^{-\alpha / 2},
$$

see, e.g., [18, p. 1581]

2.5. Approximation of the Distribution of a Scalar SDE. We consider a scalar autonomous SDE

$$
\begin{aligned}
\mathrm{d} X(t) & =a(X(t)) \mathrm{d} t+b(X(t)) \mathrm{d} W(t), \quad t \in[0,1], \\
X(0) & =x_{0}
\end{aligned}
$$

with a deterministic initial value $x_{0} \in \mathbb{R}$ and a scalar Brownian motion $W$. Both, the drift coefficient $a: \mathbb{R} \rightarrow \mathbb{R}$ and the diffusion coefficient $b: \mathbb{R} \rightarrow \mathbb{R}$ are assumed to be differentiable with bounded and Lipschitz continuous derivatives. This yields, in particular,

$$
\mathrm{E} \sup _{t \in[0,1]}|X(t)|^{2}<\infty .
$$

Furthermore, we assume that $b\left(x_{0}\right) \neq 0$ in order to exclude the case of a deterministic equation.

At first, we consider the random bit approximation of marginal distributions of $X$. To this end we consider the Milstein scheme based on the equidistant points

$$
t_{k}=t_{k, m}=k / m, \quad k=0, \ldots, m,
$$

where $m \in \mathbb{N}$. In terms of the normalized increments

$$
Y_{k}=Y_{k, m}=m^{1 / 2} \cdot\left(W\left(t_{k}\right)-W\left(t_{k-1}\right)\right)
$$

the scheme reads as

$$
\begin{aligned}
& X_{m}\left(t_{0}\right)=x_{0}, \\
& \begin{aligned}
X_{m}\left(t_{k}\right)=X_{m}\left(t_{k-1}\right)+a\left(X_{m}\left(t_{k-1}\right)\right) \cdot m^{-1}+b\left(X_{m}\left(t_{k-1}\right)\right) \cdot m^{-1 / 2} \cdot Y_{k} \\
\quad+\frac{1}{2} \cdot\left(b \cdot b^{\prime}\right)\left(X_{m}\left(t_{k-1}\right)\right) \cdot m^{-1} \cdot\left(Y_{k}^{2}-1\right),
\end{aligned}
\end{aligned}
$$

where $k=1, \ldots, m$. The following result is well known, see, e.g., [20, Thm. 1.2.4].

Lemma 3. We have

$$
\left(\mathrm{E}\left(\max _{k=1, \ldots, m}\left|X\left(t_{k}\right)-X_{m}\left(t_{k}\right)\right|^{2}\right)\right)^{1 / 2} \preceq m^{-1} .
$$


Let $q \in \mathbb{N}$. The approximation

$$
Y_{k}^{(q)}=Y_{k, m}^{(q)}=\Phi^{-1} \circ T^{(q)} \circ \Phi\left(Y_{k}\right)
$$

of the normalized increments, cf. (4), leads to the random bit Milstein scheme

$$
\begin{aligned}
X_{m}^{(q)}\left(t_{0}\right) & =x_{0} \\
X_{m}^{(q)}\left(t_{k}\right) & =X_{m}^{(q)}\left(t_{k-1}\right)+a\left(X_{m}^{(q)}\left(t_{k-1}\right)\right) \cdot m^{-1}+b\left(X_{m}^{(q)}\left(t_{k-1}\right)\right) \cdot m^{-1 / 2} \cdot Y_{k}^{(q)} \\
& \quad+\frac{1}{2} \cdot\left(b \cdot b^{\prime}\right)\left(X_{m}^{(q)}\left(t_{k-1}\right)\right) \cdot m^{-1} \cdot\left(\left(Y_{k}^{(q)}\right)^{2}-1\right),
\end{aligned}
$$

where $k=1, \ldots, m$.

We are going to employ results from [21], which deals with the quantization problem. In the latter setting approximations $\tilde{Y}_{k}^{(q)}$ to $Y_{k}$ with distributions in $\mathfrak{F}(\mathbb{R}, q)$ and error of order $2^{-q}$ are available, see Remark 7. However, the method of proof for Lemma 3 from [21] is immediately applicable in the present setting of random bit approximation, where we rely on Theorem 11.

Lemma 4 (Cf. [21, Lemma 3]). We have

$$
\left(\mathrm{E}\left(\max _{k=1, \ldots, m}\left|X_{m}\left(t_{k}\right)-X_{m}^{(q)}\left(t_{k}\right)\right|^{2}\right)\right)^{1 / 2} \preceq m^{-1}+2^{-q / 2} \cdot q^{-1 / 2}
$$

uniformly in $m, q \in \mathbb{N}$.

Remark 10. Let $\nu_{m}^{(q)}$ denote the joint distribution of $X_{m}^{(q)}\left(t_{1}\right), \ldots, X_{m}^{(q)}\left(t_{m}\right)$ and let $\nu$ denote the corresponding marginal distribution of $X$. Consider the supremum norm on $V=\mathbb{R}^{m}$. The joint distribution of $Y_{1}^{(q)}, \ldots, Y_{m}^{(q)}$ belongs to $\mathfrak{U}\left(\mathbb{R}^{m}, m q\right)$, and therefore $\nu_{m}^{(q)} \in \mathfrak{R}\left(\mathbb{R}^{m}, m q\right)$. Lemmata 3 and 4 yield

$$
\operatorname{rbit}(\nu, m q) \leq\left(\mathrm{E}\left(\max _{k=1, \ldots, m}\left|X\left(t_{k}\right)-X_{m}^{(q)}\left(t_{k}\right)\right|^{2}\right)\right)^{1 / 2} \preceq m^{-1}+2^{-q / 2} \cdot q^{-1 / 2} .
$$

Now we turn to the random bit approximation of the distribution of $X$ on the space $L_{2}=$ $L_{2}([0,1])$. We employ a piecewise linear interpolation together with a local refinement of the Milstein approximation on each of the subintervals $\left[t_{k-1}, t_{k}\right]$. To this end we consider the Brownian bridges

$$
B_{k}(t)=B_{k, m}(t)=m^{1 / 2} \cdot\left(W\left(t_{k-1}+t / m\right)-W\left(t_{k-1}\right)\right)-t \cdot Y_{k}, \quad t \in[0,1],
$$

and we define

$$
\begin{array}{r}
\bar{X}_{m}(t)=\left(t-t_{k-1}\right) \cdot m \cdot X_{m}\left(t_{k}\right)+\left(t_{k}-t\right) \cdot m \cdot X_{m}\left(t_{k-1}\right) \\
+b\left(X_{m}\left(t_{k-1}\right)\right) \cdot m^{-1 / 2} \cdot B_{k}\left(\left(t-t_{k-1}\right) \cdot m\right)
\end{array}
$$

where $t \in\left[t_{k-1}, t_{k}\right]$ and $k=1, \ldots, m$.

Lemma 5 ([21, Lemma 4]). We have

$$
\sup _{t \in[0,1]}\left(\mathrm{E}\left|X(t)-\bar{X}_{m}(t)\right|^{2}\right)^{1 / 2} \preceq m^{-1} .
$$


Finally we choose $\boldsymbol{p}(\ell)$ according to (14), and we define $X_{m}^{(q, \ell)}$ analogously to $\bar{X}_{m}$, replacing $X_{m}$ by $X_{m}^{(q)}$ and $B_{k}$ by $B_{k}^{(\ell, p(\ell))}=B_{k, m}^{(\ell, p(\ell))}$. This leads to

$$
\begin{aligned}
X_{m}^{(q, \ell)}(t)=(t & \left.-t_{k-1}\right) \cdot m \cdot X_{m}^{(q)}\left(t_{k}\right)+\left(t_{k}-t\right) \cdot m \cdot X_{m}^{(q)}\left(t_{k-1}\right) \\
& +b\left(X_{m}^{(q)}\left(t_{k-1}\right)\right) \cdot m^{-1 / 2} \cdot B_{k}^{(\ell, p(\ell))}\left(\left(t-t_{k-1}\right) \cdot m\right),
\end{aligned}
$$

where $t \in\left[t_{k-1}, t_{k}\right]$ and $k=1, \ldots, m$. The distribution of $B_{k}^{(\ell, \boldsymbol{p}(\ell))}$ belongs to $\mathfrak{U}\left(L_{2},|\boldsymbol{p}(\ell)|\right)$, and $|\boldsymbol{p}(\ell)|=2^{\ell+2}-2 \ell-4$, see Theorem 2. Therefore the distribution of $X_{m}^{(q, \ell)}$ belongs to $\mathfrak{R}\left(L_{2}, c(m, q, \ell)\right)$, where

$$
c(m, q, \ell)=m \cdot\left(q+2^{\ell+2}-2 \ell-4\right) .
$$

Lemma 6. We have

$$
\left(\mathrm{E}\left\|X-X_{m}^{(q, \ell)}\right\|_{L_{2}}^{2}\right)^{1 / 2} \preceq m^{-1}+2^{-q / 2} \cdot q^{-1 / 2}+m^{-1 / 2} \cdot 2^{-\ell / 2}
$$

uniformly in $m, q, \ell \in \mathbb{N}$.

Proof. We closely follow the proof of [21, Lemma 5], and we write $\boldsymbol{p}$ instead of $\boldsymbol{p}(\ell)$ to simplify the notation. Due to Lemma 5 it suffices to analyze $\bar{X}_{m}-X_{m}^{(q, \ell)}$. This difference is split up into

$$
\bar{X}_{m}-X_{m}^{(q, \ell)}=U_{1}+U_{2}+U_{3}
$$

where

$$
U_{1}(t)=\left(t-t_{k-1}\right) \cdot m \cdot\left(X_{m}\left(t_{k}\right)-X_{m}^{(q)}\left(t_{k}\right)\right)+\left(t_{k}-t\right) \cdot m \cdot\left(X_{m}\left(t_{k-1}\right)-X_{m}^{(q)}\left(t_{k-1}\right)\right),
$$

as well as

$$
U_{2}(t)=\left(b\left(X_{m}\left(t_{k-1}\right)\right)-b\left(X_{m}^{(q)}\left(t_{k-1}\right)\right)\right) \cdot m^{-1 / 2} \cdot B_{k}\left(\left(t-t_{k-1}\right) \cdot m\right)
$$

and

$$
U_{3}(t)=b\left(X_{m}^{(q)}\left(t_{k-1}\right)\right) \cdot m^{-1 / 2} \cdot\left(B_{k}\left(\left(t-t_{k-1}\right) \cdot m\right)-B_{k}^{(\ell, p)}\left(\left(t-t_{k-1}\right) \cdot m\right)\right)
$$

for $t \in\left[t_{k-1}, t_{k}\right]$.

Put

$$
\Delta=\Delta_{m}^{(q)}=\max _{k=1, \ldots, m}\left|X_{m}\left(t_{k}\right)-X_{m}^{(q)}\left(t_{k}\right)\right|
$$

and observe that

$$
\mathrm{E}\left(\Delta^{2}\right) \preceq m^{-2}+2^{-q} \cdot q^{-1}
$$

see Lemma 4. Clearly $\left|U_{1}(t)\right| \leq \Delta$, and therefore

$$
\mathrm{E}\left\|U_{1}\right\|_{L_{2}}^{2} \preceq \mathrm{E}\left(\Delta^{2}\right) .
$$

The Lipschitz continuity of $b$ yields

$$
\left|U_{2}(t)\right| \preceq \Delta \cdot m^{-1 / 2} \cdot B_{k}\left(\left(t-t_{k-1}\right) \cdot m\right)
$$

for $t \in\left[t_{k-1}, t_{k}\right]$ and $k=1, \ldots, m$. Moreover,

$$
\mathrm{E}\left\|B_{k}\left(\left(\cdot-t_{k-1}\right) \cdot m\right)\right\|_{L_{2}\left(\left[t_{k-1}, t_{k}\right]\right)}^{2} \asymp m^{-1} .
$$

We use the independence of $\Delta$ and $\left(B_{1}, \ldots, B_{m}\right)$ to conclude that

$$
\mathrm{E}\left\|U_{2}\right\|_{L_{2}}^{2} \preceq \mathrm{E}\left(\Delta^{2}\right) \cdot m^{-1} \cdot \sum_{k=1}^{m} \mathrm{E}\left\|B_{k}\left(\left(\cdot-t_{k-1}\right) \cdot m\right)\right\|_{L_{2}\left(\left[t_{k-1}, t_{k}\right]\right)}^{2} \asymp m^{-1} \cdot \mathrm{E}\left(\Delta^{2}\right) .
$$


Altogether

$$
\mathrm{E}\left\|U_{1}+U_{2}\right\|_{L_{2}}^{2} \preceq m^{-2}+2^{-q} \cdot q^{-1} .
$$

It remains to consider the term $U_{3}$. From (22) and (23) we get

$$
\sup _{m \in \mathbb{N}, q \in \mathbb{N}} \mathrm{E} \max _{k=1, \ldots, m}\left|X_{m}^{(q)}\left(t_{k}\right)\right|^{2}<\infty .
$$

Moreover,

$$
\begin{aligned}
\mathrm{E}\left\|B_{k}\left(\left(\cdot-t_{k-1}\right) \cdot m\right)-B_{k}^{(\ell, \boldsymbol{p})}\left(\left(\cdot-t_{k-1}\right) \cdot m\right)\right\|_{L_{2}\left(\left[t_{k-1}, t_{k}\right]\right)}^{2} & \asymp m^{-1} \cdot \mathrm{E}\left\|B-B^{(\ell, \boldsymbol{p})}\right\|_{L_{2}}^{2} \\
& \asymp m^{-1} \cdot 2^{-\ell},
\end{aligned}
$$

see Theorem 2. Since $b$ satisfies a linear growth condition we get

$$
\mathrm{E}\left\|U_{3}\right\|_{L_{2}}^{2} \preceq m^{-1} \cdot 2^{-\ell}
$$

from the independence of $\max _{k=1, \ldots, m}\left|X_{m}^{(q)}\left(t_{k}\right)\right|$ and $\left(B_{1}, \ldots, B_{m}\right)$.

Remark 11. Suppose that the Euler scheme, instead of the Milstein scheme, would be employed in the definition of $X_{m}^{(q, \ell)}$. Then the first term in the upper bound from Lemma 6 would change from $m^{-1}$ to $m^{-1 / 2}$, so that altogether

$$
\left(\mathrm{E}\left\|X-X_{m}^{(q, \ell)}\right\|_{L_{2}}^{2}\right)^{1 / 2} \preceq m^{-1 / 2}+2^{-q / 2} \cdot q^{-1 / 2},
$$

which does not suffice for our purposes.

Theorem 4. Let $\mu$ denote the distribution of $X$ on $L_{2}$. Then we have

$$
\operatorname{rbit}(\mu, p) \asymp \operatorname{quant}(\mu, p) \asymp p^{-1 / 2} \text {. }
$$

Furthermore, let

$$
m(\ell)=2^{\ell}, \quad q(\ell)=2 \ell,
$$

and $c(\ell)=c(m(\ell), q(\ell), \ell)$. Then we have

$$
\left(\mathrm{E}\left\|X-X_{m(\ell)}^{(q(\ell), \ell)}\right\|_{L_{2}}^{2}\right)^{1 / 2} \asymp \operatorname{rbit}(\mu, c(\ell))
$$

and

$$
c(\ell)=2^{\ell+2} \cdot\left(2^{\ell}-1\right) \asymp 2^{2 \ell} .
$$

Proof. We write $m, q$, and $\boldsymbol{p}$ instead of $m(\ell), q(\ell)$, and $\boldsymbol{p}(\ell)$, respectively, to simplify the notation. By definition,

$$
\operatorname{rbit}(\mu, c(\ell)) \leq\left(\mathrm{E}\left\|X-X_{m}^{(q, \ell)}\right\|_{L_{2}}^{2}\right)^{1 / 2}
$$

Hence we show that

$$
\left(\mathrm{E}\left\|X-X_{m}^{(q, \ell)}\right\|_{L_{2}}^{2}\right)^{1 / 2} \preceq c(\ell)^{-1 / 2}
$$

Use Lemma 6] to derive

$$
\left(\mathrm{E}\left\|X-X_{m}^{(q, \ell)}\right\|_{L_{2}}^{2}\right)^{1 / 2} \preceq 2^{-\ell}
$$

The explicit formula for $c(\ell)$ obviously holds true, and this completes the proof of the asymptotic upper bound (24).

On the other hand,

$$
\text { quant }(\mu, p) \asymp p^{-1 / 2}
$$


see [6, Thm. 1.1]; the same asymptotic result for quantization is derived in [3, 19] under stronger assumptions.

\section{Random Bit Quadrature with respect to Gaussian Measures}

As in Sections 2.3 and 2.4 we consider a centered Gaussian random element $X$ that takes values in an infinite-dimensional separable Hilbert space $\left(H,\|\cdot\|_{H}\right)$. We define and analyze algorithms that use random bits for the approximation of

$$
S(f)=\mathrm{E}(f(X))
$$

for functionals

$$
f: H \rightarrow \mathbb{R}
$$

that are Lipschitz continuous with Lipschitz constant one, i.e.,

$$
|f(x)-f(y)| \leq\|x-y\|_{H}
$$

for all $x, y \in H$. For comparison we also consider algorithms that may use uniformly distributed random numbers from $[0,1]$ instead of random bits.

Let $\operatorname{Lip}_{1}$ denote the corresponding class of all such functionals $f$, and let $\mu$ denote the distribution of $X$ on $H$. Of course, the output $A(f)$ of a randomized (Monte Carlo) algorithm $A$ on input $f \in \operatorname{Lip}_{1}$ is a random quantity, and therefore the worst case error of $A$ on the class $\operatorname{Lip}_{1}$ is defined by

$$
e\left(A, \operatorname{Lip}_{1}, \mu\right)=\sup _{f \in \operatorname{Lip}_{1}}\left(\mathrm{E}|S(f)-A(f)|^{2}\right)^{1 / 2}
$$

Consider any increasing sequence

$$
H_{1} \subseteq H_{2} \subseteq \ldots
$$

of finite-dimensional subspaces of $H$ such that $\operatorname{dim} H_{n}=n$ for $n \in \mathbb{N}$, and put $\tilde{H}=$ $\bigcup_{n=1}^{\infty} H_{n}$ as well as $H_{0}=\emptyset$. We suppose that a randomized algorithm may evaluate any functional $f \in \operatorname{Lip}_{1}$ at any point $x \in \tilde{H}$ at cost $n$, if $x \in H_{n} \backslash H_{n-1}$. Furthermore, algorithms are assumed to perform arithmetic operations with real numbers exactly and to evaluate elementary functions at unit cost. Finally, the algorithms have access to a random number generator at cost one per call, and here we distinguish two cases. If the generator provides random bits, we use the term of a restricted Monte Carlo algorithm. Otherwise, if the generator provides random numbers from $[0,1]$, the algorithm is called a Monte Carlo algorithm.

By $\operatorname{cost}(A, f)$ we denote the cost for applying the randomized algorithm $A$ to the functional $f$, which is defined as the sum of the cost associated to every instruction that is carried out. Observe that $\operatorname{cost}(A, f)$ is a random quantity, analogously to $A(f)$, and therefore the worst case cost of $A$ on the class $\operatorname{Lip}_{1}$ is defined by

$$
\operatorname{cost}\left(A, \operatorname{Lip}_{1}\right)=\sup _{f \in \operatorname{Lip}_{1}} \mathrm{E}(\operatorname{cost}(A, f)) .
$$

This cost model, which is called variable subspace sampling, is appropriate for quadrature problems on infinite-dimensional spaces, see [3] for details and for the mild measurability assumptions involved. The latter are obviously satisfied for the specific algorithms to be constructed below. 
We are particularly interested in multilevel Monte Carlo algorithms, see [12] for a survey. At first we consider the setting from Section 2.4 with the natural choice of subspaces

$$
H_{n}=\operatorname{span}\left\{e_{1}, \ldots, e_{n}\right\} .
$$

It follows, in particular, that $\tilde{H}$ is a dense subspace of the support of $\mu$. In the present setting of a quadrature problem with random bits we construct a multilevel algorithm as follows. Let $L \in \mathbb{N}$ be the maximal level of the multilevel algorithm. On every level $\ell=2, \ldots, L$ the algorithm involves a fine and a coarse approximation that are based on the first $m=2^{\ell}$ and $m=2^{\ell-1}$ terms, respectively, of the Karhunen-Loève expansion of $X$. On level $\ell=1$ we only consider the fine approximation with $m=2$ terms. The bit numbers that are used to approximate the random coefficients $Y_{i}$ of $X$ are chosen according to Theorem 3. In this way we have two dimensions of discretization: the truncation level for the Karhunen-Loève expansion and the bit numbers for the approximation of the random coefficients.

Let $N_{1}, \ldots, N_{L} \in \mathbb{N}$ be the replication numbers on the levels $1, \ldots, L$, and let $X_{\ell, j}$ with $\ell=1, \ldots, L$ and $j=1, \ldots, N_{\ell}$ denote independent copies of $X$. Recall the definition of $\boldsymbol{p}(m) \in \mathbb{N}^{m}$ and $X^{(m, p(m))}$ according to Theorem 3 . We study the multilevel Monte Carlo algorithm

$$
A^{L, N_{1}, \ldots, N_{L}}(f)=\frac{1}{N_{1}} \sum_{j=1}^{N_{1}} f\left(X_{1, j}^{(2, \boldsymbol{p}(2))}\right)+\sum_{\ell=2}^{L} \frac{1}{N_{\ell}} \sum_{j=1}^{N_{\ell}}\left(f\left(X_{\ell, j}^{\left(2^{\ell}, \boldsymbol{p}\left(2^{\ell}\right)\right)}\right)-f\left(X_{\ell, j}^{\left(2^{\ell-1}, \boldsymbol{p}\left(2^{\ell-1}\right)\right)}\right)\right) .
$$

At first we show that this algorithm only requires $\sum_{\ell=1}^{L} N_{\ell} \cdot\left|\boldsymbol{p}\left(2^{\ell}\right)\right|$ calls to the random number generator for random bits. Since the $X_{\ell, j}$ are independent copies of $X$ and since $p_{i}\left(2^{\ell-1}\right) \leq p_{i}\left(2^{\ell}\right)$ for $i=1, \ldots, 2^{\ell-1}$ and $\ell \in \mathbb{N}$, it suffices to show that the joint distribution of $X^{(m, \boldsymbol{p})}$ and $X^{(\tilde{m}, \tilde{\boldsymbol{p}})}$ can be simulated using $|\boldsymbol{p}|$ random bits, where $1 \leq \tilde{m} \leq m$ as well as $\boldsymbol{p} \in \mathbb{N}^{m}$ and $\tilde{\boldsymbol{p}} \in \mathbb{N}^{\tilde{m}}$ with $\tilde{p}_{i} \leq p_{i}$ for all $i=1, \ldots, \tilde{m}$. Recall that $X=\sum_{i=1}^{\infty} \lambda_{i}^{1 / 2} \cdot Y_{i} \cdot e_{i}$. For $i=1, \ldots, m$ define

$$
U_{i}=T^{\left(p_{i}\right)} \circ \Phi\left(Y_{i}\right)
$$

By definition we have

$$
\begin{aligned}
X^{(m, p)} & =\sum_{i=1}^{m} \lambda_{i}^{1 / 2} \cdot Y_{i}^{\left(p_{i}\right)} \cdot e_{i}=\sum_{i=1}^{m} \lambda_{i}^{1 / 2} \cdot \Phi^{-1} \circ T^{\left(p_{i}\right)} \circ \Phi\left(Y_{i}\right) \cdot e_{i} \\
& =\sum_{i=1}^{m} \lambda_{i}^{1 / 2} \cdot \Phi^{-1}\left(U_{i}\right) \cdot e_{i} .
\end{aligned}
$$

Since $\tilde{p}_{i} \leq p_{i}$ for $i=1, \ldots, \tilde{m}$, we get $T^{\left(\tilde{p}_{i}\right)}=T^{\left(\tilde{p}_{i}\right)} \circ T^{\left(p_{i}\right)}$ and therefore

$$
\begin{aligned}
X^{(\tilde{m}, \tilde{\boldsymbol{p}})} & =\sum_{i=1}^{\tilde{m}} \lambda_{i}^{1 / 2} \cdot Y_{i}^{\left(\tilde{p}_{i}\right)} \cdot e_{i}=\sum_{i=1}^{\tilde{m}} \lambda_{i}^{1 / 2} \cdot \Phi^{-1} \circ T^{\left(\tilde{p}_{i}\right)} \circ \Phi\left(Y_{i}\right) \cdot e_{i} \\
& =\sum_{i=1}^{\tilde{m}} \lambda_{i}^{1 / 2} \cdot \Phi^{-1} \circ T^{\left(\tilde{p}_{i}\right)}\left(U_{i}\right) \cdot e_{i} .
\end{aligned}
$$

Combining (25) and (26) with the fact that $U_{i}$ is uniformly distributed on $D^{\left(p_{i}\right)}$ and $U_{1}, \ldots, U_{m}$ are independent, we conclude that the joint distribution of $X^{(m, p)}$ and $X^{(\tilde{m}, \tilde{\boldsymbol{p}})}$ can be simulated using $|\boldsymbol{p}|$ random bits. 
Theorem 5. Let $\mu$ denote the distribution of the Gaussian random element $X$ on $H$, assume that (19) is satisfied, and let $\varepsilon \in] 0, \exp (-2)]$. Choose

$$
L=L(\varepsilon)=\left\lceil\frac{2}{\beta-1} \cdot \log _{2}(z(\varepsilon))\right\rceil
$$

with

$$
z=z(\varepsilon)=1+\varepsilon^{-1} \cdot\left(\ln \left(\varepsilon^{-1}\right)\right)^{-\alpha / 2}
$$

as well as

$$
N_{\ell}=N_{\ell}(\varepsilon)=\left\lceil 2^{-\ell \beta / 2} \cdot \ell^{-\alpha / 2} \cdot K(\varepsilon)\right\rceil
$$

for $\ell=1, \ldots, L$, where

$$
K=K(\varepsilon)=\varepsilon^{-\max (2, \beta /(\beta-1))} \cdot \begin{cases}1, & \text { if } \beta>2, \\ \left(\ln \left(\varepsilon^{-1}\right)\right)^{\max (0,1-\alpha / 2)}, & \text { if } \beta=2 \wedge \alpha \neq 2, \\ \ln \left(\ln \left(\varepsilon^{-1}\right)\right), & \text { if } \beta=2 \wedge \alpha=2, \\ \left(\ln \left(\varepsilon^{-1}\right)\right)^{\frac{\alpha}{2(1-\beta)}}, & \text { if } \beta<2 .\end{cases}
$$

Then the random bit multilevel algorithm $A^{(\varepsilon)}=A^{L, N_{1}, \ldots, N_{L}}$ satisfies

$$
e\left(A^{(\varepsilon)}, \operatorname{Lip}_{1}, \mu\right) \preceq \varepsilon
$$

and

$$
\operatorname{cost}\left(A^{(\varepsilon)}, \operatorname{Lip}_{1}\right) \asymp \varepsilon^{-\max (2,2 /(\beta-1))} \cdot \begin{cases}1, & \text { if } \beta>2, \\ \left(\ln \left(\varepsilon^{-1}\right)\right)^{\max (0,2-\alpha)}, & \text { if } \beta=2 \wedge \alpha \neq 2, \\ \left(\ln \left(\ln \left(\varepsilon^{-1}\right)\right)^{2},\right. & \text { if } \beta=2 \wedge \alpha=2, \\ \left(\ln \left(\varepsilon^{-1}\right)\right)^{\frac{\alpha}{1-\beta}}, & \text { if } \beta<2 .\end{cases}
$$

Proof. At first we consider the cost of $A^{(\varepsilon)}$. Theorem 3 implies $\left|\boldsymbol{p}\left(2^{\ell}\right)\right| \asymp 2^{\ell}$, so that

$$
\operatorname{cost}\left(A^{(\varepsilon)}, \operatorname{Lip}_{1}\right) \asymp \sum_{\ell=1}^{L} 2^{\ell} \cdot N_{\ell} .
$$

In fact the number of calls to the random number generator for random bits is of this order, see the discussion directly before Theorem 5, and the same holds true for the number of arithmetic operations as well as for the cost associated to the evaluation of $f$. Observe that

$$
L \asymp \ln \left(\varepsilon^{-1}\right)
$$

and

$$
2^{L} \asymp z^{\frac{2}{\beta-1}}
$$

Therefore

$$
2^{-L \beta / 2} \cdot L^{-\alpha / 2} \asymp z^{\frac{\beta}{1-\beta}} \cdot L^{-\alpha / 2} \asymp \varepsilon^{\frac{\beta}{\beta-1}} \cdot\left(\ln \left(\varepsilon^{-1}\right)\right)^{\frac{\alpha}{2(\beta-1)}} .
$$

We conclude that

$$
2^{-\ell \beta / 2} \cdot \ell^{-\alpha / 2} \cdot K \succeq 2^{-L \beta / 2} \cdot L^{-\alpha / 2} \cdot K \succeq 1,
$$

and consequently that

$$
N_{\ell} \asymp 2^{-\ell \beta / 2} \cdot \ell^{-\alpha / 2} \cdot K
$$


both hold uniformly in $\varepsilon$ and $\ell=1, \ldots, L$. It follows that

$$
\operatorname{cost}\left(A^{(\varepsilon)}, \operatorname{Lip}_{1}\right) \asymp K \cdot \sum_{\ell=1}^{L} 2^{\ell(1-\beta / 2)} \cdot \ell^{-\alpha / 2} .
$$

Furthermore

$$
\sum_{\ell=1}^{L} 2^{\ell(1-\beta / 2)} \cdot \ell^{-\alpha / 2} \asymp \begin{cases}1, & \text { if } \beta>2, \\ L^{\max (0,1-\alpha / 2)}, & \text { if } \beta=2 \wedge \alpha \neq 2, \\ \ln (1+L), & \text { if } \beta=2 \wedge \alpha=2, \\ 2^{L(1-\beta / 2)} \cdot L^{-\alpha / 2}, & \text { if } \beta<2 .\end{cases}
$$

Since

$$
2^{L(1-\beta / 2)} \cdot L^{-\alpha / 2} \asymp z^{\frac{2-\beta}{\beta-1}} \cdot L^{-\alpha / 2} \asymp \varepsilon^{-\frac{2-\beta}{\beta-1}} \cdot\left(\ln \left(\varepsilon^{-1}\right)\right)^{\frac{\alpha}{2(1-\beta)}},
$$

we obtain

$$
\sum_{\ell=1}^{L} 2^{\ell(1-\beta / 2)} \cdot \ell^{-\alpha / 2} \asymp \begin{cases}1, & \text { if } \beta>2, \\ \left(\ln \left(\varepsilon^{-1}\right)\right)^{\max (0,1-\alpha / 2)}, & \text { if } \beta=2 \wedge \alpha \neq 2, \\ \ln \left(\ln \left(\varepsilon^{-1}\right)\right), & \text { if } \beta=2 \wedge \alpha=2, \\ \varepsilon^{-\frac{2-\beta}{\beta-1}} \cdot\left(\ln \left(\varepsilon^{-1}\right)\right)^{\frac{\alpha}{2(1-\beta)}}, & \text { if } \beta<2 .\end{cases}
$$

Together with (27) this yields the asymptotic estimate for $\operatorname{cost}\left(A^{(\varepsilon)}, \operatorname{Lip}_{1}\right)$ as claimed.

It remains to establish the asymptotic upper bound for the error of $A^{(\varepsilon)}$. Observe that

$$
\left|S(f)-\mathrm{E}\left(A^{(\varepsilon)}(f)\right)\right|=\left|\mathrm{E}(f(X))-\mathrm{E}\left(f\left(X^{\left(2^{L}, \boldsymbol{p}\left(2^{L}\right)\right)}\right)\right)\right| \leq \mathrm{E}\left\|X-X^{\left(2^{L}, \boldsymbol{p}\left(2^{L}\right)\right)}\right\|_{H}
$$

and

$$
\begin{aligned}
\operatorname{Var}\left(f\left(X^{(2, \boldsymbol{p}(2))}\right)\right) & =\operatorname{Var}\left(f\left(X^{(2, \boldsymbol{p}(2))}\right)-f(0)\right) \\
& \leq \mathrm{E}\left|f\left(X^{(2, \boldsymbol{p}(2))}\right)-f(0)\right|^{2} \leq \mathrm{E}\left\|X^{(2, \boldsymbol{p}(2))}\right\|_{H}^{2}<\infty
\end{aligned}
$$

as well as

$$
\begin{aligned}
& \operatorname{Var}\left(f\left(X^{\left(2^{\ell}, \boldsymbol{p}\left(2^{\ell}\right)\right)}\right)-f\left(X^{\left(2^{\ell-1}, \boldsymbol{p}\left(2^{\ell-1}\right)\right)}\right)\right) \\
& \quad \leq 2 \cdot \mathrm{E}\left\|X-X^{\left(2^{\ell}, \boldsymbol{p}\left(2^{\ell}\right)\right)}\right\|_{H}^{2}+2 \cdot \mathrm{E}\left\|X-X^{\left(2^{\ell-1}, \boldsymbol{p}\left(2^{\ell-1}\right)\right)}\right\|_{H}^{2}
\end{aligned}
$$

for $\ell=2, \ldots, L$, due to the Lipschitz continuity of $f \in \operatorname{Lip}_{1}$. From Theorem 3 we hence get

$$
\sup _{f \in \operatorname{Lip}_{1}}\left|S(f)-\mathrm{E}\left(A^{(\varepsilon)}(f)\right)\right| \preceq 2^{-L(\beta-1) / 2} \cdot L^{-\alpha / 2} \asymp z^{-1} \cdot L^{-\alpha / 2} \asymp \varepsilon
$$

as well as

$$
\sup _{f \in \operatorname{Lip}_{1}} \operatorname{Var}\left(A^{(\varepsilon)}(f)\right) \preceq \sum_{\ell=1}^{L} \frac{1}{N_{\ell}} \cdot 2^{-\ell(\beta-1)} \cdot \ell^{-\alpha} \asymp K^{-1} \cdot \sum_{\ell=1}^{L} 2^{\ell(1-\beta / 2)} \cdot \ell^{-\alpha / 2} \asymp \varepsilon^{2},
$$

see (28), and therefore $e\left(A^{(\varepsilon)}, \operatorname{Lip}_{1}, \mu\right) \preceq \varepsilon$ as claimed.

The same analysis applies to the setting of a Brownian bridge $X$, as studied in Section 2.3. Here we employ a multilevel algorithm with the first $2^{\ell}-1$ and $2^{\ell-1}-1$ terms, respectively, of the Lévy-Ciesielski representation of $X$ for levels $\ell \geq 2$, and with only the first term for level $\ell=1$. Furthermore, the bit numbers are chosen according to Theorem 2. Theorem 5 holds true also in this case with $\beta=2$ and $\alpha=0$. 
In order to analyze the optimality of the random bit multilevel algorithm we consider the $n$-th minimal error for the random bit quadrature problem, which is defined by $e_{n}^{\text {res }}\left(\operatorname{Lip}_{1}, \mu\right)=\inf \left\{e\left(A, \operatorname{Lip}_{1}, \mu\right): A\right.$ restricted Monte Carlo algorithm, $\left.\operatorname{cost}\left(A, \operatorname{Lip}_{1}\right) \leq n\right\}$ for $n \in \mathbb{N}$. For comparison we also consider

$$
e_{n}\left(\operatorname{Lip}_{1}, \mu\right)=\inf \left\{e\left(A, \operatorname{Lip}_{1}, \mu\right): A \text { Monte Carlo algorithm, } \operatorname{cost}\left(A, \operatorname{Lip}_{1}\right) \leq n\right\}
$$

Corollary 1. The n-th minimal error of restricted Monte Carlo algorithms satisfies

$$
e_{n}^{\mathrm{res}}\left(\operatorname{Lip}_{1}, \mu\right) \preceq n^{-\min (1 / 2,(\beta-1) / 2)} . \begin{cases}1, & \text { if } \beta>2, \\ (\ln (n))^{\max (0,1-\alpha / 2)}, & \text { if } \beta=2 \wedge \alpha \neq 2, \\ \ln (\ln (n)), & \text { if } \beta=2 \wedge \alpha=2, \\ (\ln (n))^{-\alpha / 2}, & \text { if } \beta<2 .\end{cases}
$$

The n-th minimal error of Monte Carlo algorithms satisfies

$$
n^{-(\beta-1) / 2} \cdot(\ln (n))^{-\alpha / 2} \preceq e_{n}\left(\operatorname{Lip}_{1}, \mu\right)
$$

if $\beta \leq 2$, and

$$
\limsup _{n \rightarrow \infty}\left(e_{n}\left(\operatorname{Lip}_{1}, \mu\right) \cdot\left(n^{1 / 2} \cdot(\ln (n))^{(1+\beta) / 2} \cdot(\ln (\ln (n)))^{\alpha / 2}\right)\right)>0
$$

if $\beta>2$.

Proof. The first claim follows directly from Theorem 5. For the proof of the second claim we consider the small ball function

$$
\phi(\varepsilon)=-\ln \left(\mu\left(\left\{v \in H:\|v\|_{H} \leq \varepsilon\right\}\right)\right)
$$

of $\mu$, where $\varepsilon \in] 0, \infty[$. From [15, Proposition 4.3] or [16, Proposition 11.3] and (19) we get

$$
\phi(\varepsilon) \asymp \varepsilon^{-2 /(\beta-1)} \cdot\left(\ln \left(\varepsilon^{-1}\right)\right)^{-\alpha /(\beta-1)}
$$

for $\varepsilon \in] 0, \exp (-1)]$. Combining this with [3, Thm. 10] yields the second claim.

Remark 12. In the case $\beta<2$, Corollary 1 provides sharp upper and lower bounds, up to multiplicative constants, for the $n$-minimal errors with $e_{n}^{\mathrm{res}}\left(\operatorname{Lip}_{1}, \mu\right) \asymp e_{n}\left(\operatorname{Lip}_{1}, \mu\right)$. For $\beta=2$ we have sharp bounds up to logarithmic factors, and, in particular, a superiority of Monte Carlo algorithms over restricted Monte Carlo may at most be present on the level of such logarithmic factors. For $\beta>2$ the bounds are sharp only up to logarithmic factors and up to the presence of a limsup in the lower bound for $e_{n}\left(\operatorname{Lip}_{1}, \mu\right)$. Note that for many infinite-dimensional quadrature problems the asymptotic behavior of minimal errors is only known up to logarithmic factors.

The lower bounds from Corollary 1 are also true, if every random bit algorithm is allowed to choose the hierarchy of subspaces $H_{n}$ on its own and, roughly speaking, without any restriction on the randomness that algorithms are allowed to use. Furthermore, the general situation of a Banach space is considered in [3]. The upper bound from Corollary 1$]$ improves the upper bound from [3, Thm. 10] in terms of powers of $\ln (n)$ or $\ln (\ln (n))$, if $\beta \neq 2$ or $\alpha \geq 1$; the bounds do coincide in the remaining cases. For simplicity of the presentation we omit the details; instead we refer to [3]. 
Appendix A. Asymptotic Properties of $\Phi$ And $\Phi^{-1}$

We derive some asymptotic properties of the distribution function $\Phi$ and the inverse distribution function $\Phi^{-1}$ of the standard normal distribution. if

In the sequel, we write $f(x) \approx g(x)$ as $x \rightarrow \infty$ for two functions $f, g:] a, \infty[\rightarrow \mathbb{R} \backslash\{0\}$

$$
\lim _{x \rightarrow \infty} f(x) / g(x)=1 .
$$

Analogously we define $f(x) \approx g(x)$ as $x \searrow 0$ and $x \nearrow 1$, respectively.

We make use of

$$
1-\Phi(x) \approx x^{-1} \cdot \varphi(x)
$$

as $x \rightarrow \infty$, which is well known and follows, e.g., from L'Hôpital's Rule.

Lemma 7. We have

$$
\Phi^{-1}\left(1-2^{-p}\right) \approx \sqrt{\ln 4} \cdot p^{1 / 2}
$$

as $p \rightarrow \infty$.

Proof. With $c=\sqrt{\ln 4}$ and $x=1-2^{-p}$, i.e., $p=-\log _{2}(1-x)$, we have to show that

$$
\Phi^{-1}(x) \approx c \cdot\left(-\log _{2}(1-x)\right)^{1 / 2}
$$

as $x \nearrow 1$. Setting $x=\Phi(y)$ this is equivalent to

$$
y \approx c \cdot\left(-\log _{2}(1-\Phi(y))\right)^{1 / 2}
$$

as $y \rightarrow \infty$. Due to (29) and L'Hôpital's Rule we get in fact

$$
\left(-\log _{2}(1-\Phi(y))\right)^{1 / 2} \approx y / c
$$

as $y \rightarrow \infty$.

Lemma 8. Let $h$ be defined by (11). Then we have

$$
2^{-p} p \cdot h\left(\Phi^{-1}\left(1-2^{-p}\right)\right) \approx(\sqrt{2 \pi} \ln 4)^{-1}
$$

as $p \rightarrow \infty$.

Proof. Let $a=\Phi^{-1}\left(1-2^{-p}\right)$. Use L'Hôpital's Rule, or integration by parts, to verify

$$
h(a) \approx a^{-1} \cdot \exp \left(a^{2} / 2\right)
$$

as $a \rightarrow \infty$. Moreover, observe that

$$
p \approx a^{2} / \ln 4
$$

as $p \rightarrow \infty$, see Lemma [7, and note that $1-\Phi(a)=2^{-p}$. Altogether with (29), we obtain

$$
2^{-p} p \cdot h(a) \approx(1-\Phi(a)) \cdot a^{2} / \ln 4 \cdot h(a) \approx(\sqrt{2 \pi} \ln 4)^{-1}
$$

as $p \rightarrow \infty$.

Lemma 9. Let $g$ be defined by (12). Then we have

$$
g\left(\Phi^{-1}\left(1-2^{-(p+1)}\right)\right) \approx 2^{-p} \cdot p^{-1} / \ln 4
$$

as $p \rightarrow \infty$. 
Proof. Set $a=\Phi^{-1}\left(1-2^{-(p+1)}\right)$. At first we show

$$
g(a) \approx 2 \cdot(1-\Phi(a)) \cdot a^{-2}
$$

as $a \rightarrow \infty$. By (29) this reduces to showing

$$
g(a) \approx 2 \cdot \varphi(a) \cdot a^{-3}
$$

as $a \rightarrow \infty$. To this end we compute the following derivatives

$$
\begin{aligned}
g^{\prime}(a) & =-2 \cdot \int_{[a, \infty[}(x-a) \cdot \varphi(x) \mathrm{d} x, \\
g^{\prime \prime}(a) & =2 \cdot \int_{[a, \infty[} \varphi(x) \mathrm{d} x, \\
g^{\prime \prime \prime}(a) & =-2 \cdot \varphi(a)
\end{aligned}
$$

as well as

$$
\begin{aligned}
\frac{d}{d a}\left(\varphi(a) \cdot a^{-3}\right) & =-\varphi(a) \cdot a^{-2}-3 \cdot \varphi(a) \cdot a^{-4} \\
\frac{d}{d a}\left(-2 \cdot \varphi(a) \cdot a^{-2}\right) & =2 \cdot \varphi(a) \cdot a^{-1}+4 \cdot \varphi(a) \cdot a^{-3} \\
\frac{d}{d a}\left(2 \cdot \varphi(a) \cdot a^{-1}\right) & =-2 \cdot \varphi(a)-2 \cdot \varphi(a) \cdot a^{-2} .
\end{aligned}
$$

Using three times L'Hôpital's Rule yields

$$
\begin{aligned}
\lim _{a \rightarrow \infty} \frac{g(a)}{2 \cdot \varphi(a) \cdot a^{-3}} & =\lim _{a \rightarrow \infty} \frac{g^{\prime}(a)}{-2 \cdot \varphi(a) \cdot a^{-2}} \\
& =\lim _{a \rightarrow \infty} \frac{g^{\prime \prime}(a)}{2 \cdot \varphi(a) \cdot a^{-1}} \\
& =\lim _{a \rightarrow \infty} \frac{g^{\prime \prime \prime}(a)}{-2 \cdot \varphi(a)}=1 .
\end{aligned}
$$

Finally, having (30) at hand, Lemma 7 finishes the proof.

Lemma 10. For $0<x<1$ define

$$
u(x)=\varphi\left(\Phi^{-1}(1-x)\right)
$$

and

$$
v(x)=x \cdot\left(\ln \left(x^{-1}\right)\right)^{1 / 2} .
$$

Then we have

$$
u(x) \approx \sqrt{2} \cdot v(x)
$$

as $x \searrow 0$.

Proof. Set $\tilde{u}(x)=u(x)^{2}$ as well as $\tilde{v}(x)=v(x)^{2}$. The derivatives read as

$$
\begin{aligned}
& \tilde{u}^{\prime}(x)=2 \cdot \varphi\left(\Phi^{-1}(1-x)\right) \cdot \Phi^{-1}(1-x), \\
& \tilde{u}^{\prime \prime}(x)=2 \cdot\left(\Phi^{-1}(1-x)\right)^{2}-2
\end{aligned}
$$

as well as

$$
\begin{aligned}
& \tilde{v}^{\prime}(x)=-2 \cdot x \cdot \ln (x)-x, \\
& \tilde{v}^{\prime \prime}(x)=-2 \cdot \ln (x)-3 .
\end{aligned}
$$


Consequently, two times L'Hôpital yields

$$
\lim _{x \rightarrow 0} \frac{\tilde{v}(x)}{\tilde{u}(x)}=\lim _{x \rightarrow 0} \frac{-2 \cdot \ln (x)-3}{2 \cdot\left(\Phi^{-1}(1-x)\right)^{2}-2}=\lim _{x \rightarrow 0} \frac{\ln \left(x^{-1}\right)}{\left(\Phi^{-1}(1-x)\right)^{2}} .
$$

Now, Lemma 7 yields

$$
\left(\Phi^{-1}(1-x)\right)^{2} \approx 2 \cdot \ln 2 \cdot \log _{2}\left(x^{-1}\right)=2 \cdot \ln \left(x^{-1}\right)
$$

as $x \searrow 0$. Altogether we obtain

$$
\tilde{v}(x) \approx \tilde{u}(x) / 2
$$

as $x \searrow 0$.

Lemma 11. We have

$$
\Phi^{-1}(b)-\Phi^{-1}(a) \succeq(b-a) \cdot(1-a)^{-1} \cdot(-\ln (1-a))^{-1 / 2}
$$

uniformly in $1 / 2<a<b<1$.

Proof. The mean value theorem yields the existence of $m \in] a, b[$ such that

$$
\Phi^{-1}(b)-\Phi^{-1}(a)=(b-a) \cdot\left(\Phi^{-1}\right)^{\prime}(m) \geq(b-a) \cdot\left(\varphi\left(\Phi^{-1}(a)\right)\right)^{-1} .
$$

The statement is now an immediate consequence of Lemma 10.

\section{ACKNOWLEDGMENT}

The authors are grateful to Steffen Omland for many valuable discussions and contributions at an early stage of this project. We thank an anonymous referee for providing us with the reference [30].

Lukas Mayer is supported by the Deutsche Forschungsgemeinschaft (DFG) within the RTG 1932 'Stochastic Models for Innovations in the Engineering Sciences'.

\section{REFERENCES}

[1] C. Brugger, C. De Schryver, N. Wehn, S. Omland, M. Hefter, K. Ritter, A. Kostiuk, R. Korn, Mixed precision multilevel Monte Carlo on hybrid computing systems, in: 2014 IEEE Conference on Computational Intelligence for Financial Engineering Economics (CIFEr), 2014, pp. 215222.

[2] J. Chevallier, Uniform decomposition of probability measures: quantization, classification, rate of convergence, arXiv:1801:02871, (2018).

[3] J. Creutzig, S. Dereich, T. Müller-Gronbach, K. Ritter, Infinite-dimensional quadrature and approximation of distributions, Found. Comput. Math. 9 (2009), 391-429.

[4] S. Dereich, High Resolution Coding of Stochastic Processes and Small Ball Probabilities, Ph.D. Thesis, FU Berlin, 2003.

[5] S. DEREICH, The coding complexity of diffusion processes under supremum norm distortion, Stochastic Process. Appl. 118 (2008), 917-937.

[6] S. DereICH, The coding complexity of diffusion processes under $L^{p}[0,1]$-norm distortion, Stochastic Process. Appl. 118 (2008), 938-951.

[7] S. DEREICH, Asymptotic formulae for coding problems and intermediate optimization problems: a review, in: Trends in Stochastic Analysis (J. Blath, P. Moerters, M. Scheutzow, eds.), Cambridge Univ. Press, Cambridge, 2009, pp. 187-232.

[8] S. Dereich, F. Fehringer, A. Matoussi, M. Scheutzow, On the link between small ball probabilities and the quantization problem for Gaussian measures on Banach spaces, J. Theor. Probab. 16 (2003), 249-265.

[9] S. Dereich, M. Scheutzow, High-resolution quantization and entropy coding for fractional Brownian motion, Electron. J. Probab. 11 (2006), 700-722. 
[10] S. Dereich, M. Scheutzow, R. Schottstedt, Constructive quantization: approximation by empirical measures, Ann. Inst. Henri Poincaré (B) 49 (2013), 1183-1203.

[11] W. Gao, P. Ye, H. Wang, Optimal error bound of restricted Monte Carlo in anisotropic Sobolev classes, Prog. Natur. Sci. 16 (2006), 588-593.

[12] M. B. Giles, Multilevel Monte Carlo methods, Acta Numer. 24 (2015), 259-328.

[13] S. Graf, H. Luschgy, Foundations of Quantization for Probability Distributions, Lecture Notes in Math. 1730, Springer, Berlin, 2000.

[14] S. Heinrich, E. Novak, H. Pfeiffer, How many random bits do we need for Monte Carlo integration?, in: MCQMC 2002 (H. Niederreiter, ed.), Springer, Berlin, 2004, pp. 27-49.

[15] A. Karol', A. Nazarov, Y. Nikitin, Small ball probabilities for Gaussian random fields and tensor products of compact operators, Trans. Amer. Math. Soc. 360 (2008), 1443-1474.

[16] M. Lifshits, Lectures on Gaussian Processes, Springer Briefs in Mathematics, Springer, Heidelberg, 2012.

[17] H. Luschgy, G. PAGÈs, Functional quantization of Gaussian processes, J. Funct. Anal. 196 (2002), $486-531$.

[18] H. Luschgy, G. PAGÈs, Sharp asymptotics for the functional quantization problem for Gaussian processes, Ann. Appl. Probab. 32 (2004), 1574-1599.

[19] H. Luschgy, G. PAGÈs, Functional quantization of a class of Brownian diffusion: a constructive approach, Stochastic Process. Appl. 116 (2006), 310-336.

[20] G. N. Milstein, M. V. Tretyakov, Stochastic Numerics for Mathematical Physics, Springer, Berlin, 2004.

[21] T. Müller-Gronbach, K. Ritter, A local refinement strategy for constructive quantization of scalar SDEs, Found. Comput. Math. 13 (2013), 1005-1033.

[22] E. NovaK, Eingeschränkte Monte Carlo Verfahren zur numerischen Integration, in: Mathematical Statistics and Applications (W. Grossman et al., eds.), Reidel, Dordrecht, 1985, pp. 269-282.

[23] E. NovaK, Deterministic and Stochastic Error Bounds in Numerical Analysis, Lecture Notes in Math. 1349, Springer, Berlin, 1988.

[24] E. NovaK, Quantum complexity of integration, J. Complexity 17 (2001), 2-16.

[25] E. Novak, H. Pfeiffer, Coin tossing algorithms for integral equations and tractability, Monte Carlo Methods Appl. 10 (2004), 491-498.

[26] S. Omland, Mixed Precision Multilevel Monte Carlo Algorithms for Reconfigurable Hardware Systems, Ph.D. Thesis, Verlag Dr. Hut, 2016.

[27] S. Omland, M. Hefter, K. Ritter, C. Brugger, C. De Schryver, N. Wehn, A. Kostiuk, Exploiting mixed-precision arithmetics in a multilevel Monte Carlo approach on FPGAs, in: FPGA Based Accelerators for Financial Applications (C. De Schryver, ed.), Springer, Cham, 2015, pp. 191220.

[28] K. Ritter, Average-Case Analysis of Numerical Problems, Lecture Notes in Math. 1733, Springer, Berlin, 2000.

[29] J. F. Traub, H. Woźniakowski, The Monte Carlo algorithm with a pseudorandom generator, Math. Comp. 58, (1992), 323-339.

[30] C. Xu, A. Berger, Best finite constrained approximations of one-dimensional probabilities, arXiv:1704:07871, (2017).

[31] P. Ye, X. Hu, Optimal integration error on anisotropic classes for restricted Monte Carlo and quantum algorithms, J. Approx. Theory 150 (2008), 24-47.

Mathematical Institute, University of Oxford, Oxford OX2 6GG, England

E-mail address: mike.giles@maths.ox.ac.uk

Fachbereich Mathematik, Technische Universität Kaiserslautern, Postfach 3049, 67653 Kaiserslautern, Germany

E-mail address: \{hefter,lmayer,ritter\}@mathematik.uni-kl.de 